\title{
Groups with a root group datum
}

\author{
Pierre-Emmanuel Caprace* Bertrand Rémy
}

\begin{abstract}
Root group data provide the abstract combinatorial framework common to all groups of Lie-type and of Kac-Moody-type. These notes intend to serve as a friendly introduction to their basic theory. We also survey some recent developments.
\end{abstract}

Keywords : root group datum, BN-pair, building, simple group, Kac-Moody group MSC 2000: 20E42, 20B07, 20F55, 51E24, 17B67

\section{Introduction}

\section{Historical overview}

Lie theory has a long and fascinating history. One of its most enthralling aspects is the gain in unity which has been acquired over the years through the contributions of many eminent figures. We try to roughly sum this up in the following paragraphs.

One of the foundational works of the theory has been the classification of simple Lie groups completed by W. Killing and É. Cartan in the first half of the 20th century: up to isomorphism, (center-free) complex simple Lie groups are in one-to-one correspondence with complex simple Lie algebras, which themselves are in one-to-one correspondence with the irreducible finite root systems. In particular, the Killing-Cartan classification highlighted five exceptional types of simple Lie groups besides the classical ones. Classical groups were then thoroughly studied and fairly well understood, mainly through case-by-case analysis [98]. Still, some nice uniform constructions of them deserve to be mentioned:

\footnotetext{
*F.N.R.S. Research Associate
} 


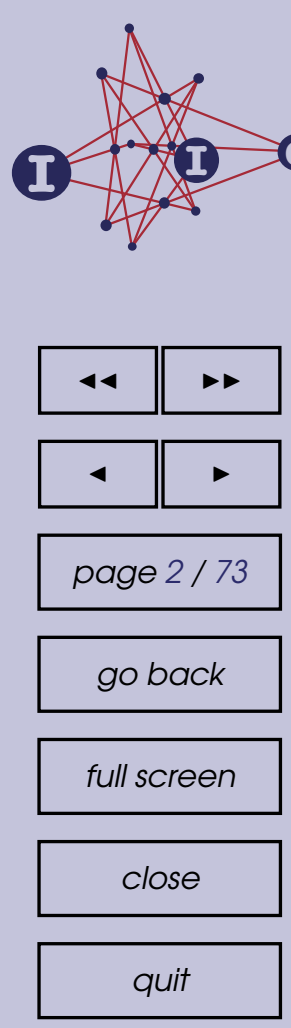

e.g., by means of algebras with involutions [99], or constructions by means of automorphism groups of some linear structures defined over an arbitrary ground field [38]. In this respect, the simple Lie groups of exceptional type were much more mysterious; analogues of them had been defined over finite fields by L. Dickson for types $E_{6}$ and $G_{2}$. A wider range of concrete realizations of exceptional groups is provided by $\mathrm{H}$. Freudenthal's work [42].

From the 1950's on, the way was paved towards a theory which would eventually embody all these groups, regardless of their type or of the underlying ground field. Two foundational papers were those of C. Chevalley [28], who constructed analogues of simple Lie groups over arbitrary fields, and of A. Borel [9], who began a systematic study of linear algebraic groups. For the sake of completeness and for the prehistory of buildings, see also [82] for an approach from the geometer's viewpoint - where "geometer" has to be understood as in J. Tits' preface to [52]. A spectacular achievement consisted in the extension by C. Chevalley of É. Cartan's classification to all simple algebraic groups over arbitrary algebraically closed fields [29]. Remarkably surprising was the fact that, once the (algebraically closed) ground field is fixed, the classification is the same as for complex Lie groups: simple algebraic groups over the given field are again in one-to-one correspondence with irreducible finite root systems.

In order to extend this correspondence to all split reductive groups over arbitrary fields, M. Demazure [36, Exp. XXI] introduced the notion of a root datum (in French: donnée radicielle), which is a refinement of the notion of root systems. These developments were especially exciting in view of the fact that most of the abstract simple groups known in the first half of the 20th century were actually related in some way to simple Lie groups.

Another further step in the unification was made by J. Tits in his seminal paper [83], where he proposed an axiomatic setting which allowed him to obtain a uniform proof of (projective) simplicity for all of these groups, as well as isotropic groups over arbitrary fields, at once. While reviewing the latter article, J. Dieudonné wrote:

"This paper goes a long way towards the realization of the hope expressed by the reviewer in 1951 that some general method be found which would give the structure of all "isotropic" classical groups without having to examine separately each type of group. It is well-known that the first breakthrough in that direction was made in the famous paper of Chevalley in 1955 [28], which bridged in a spectacular way the gap between Lie algebras and finite groups. The originality of the author has been to realize that the gist of Chevalley's arguments could be expressed in a purely group-theoretical way, namely, the existence in 


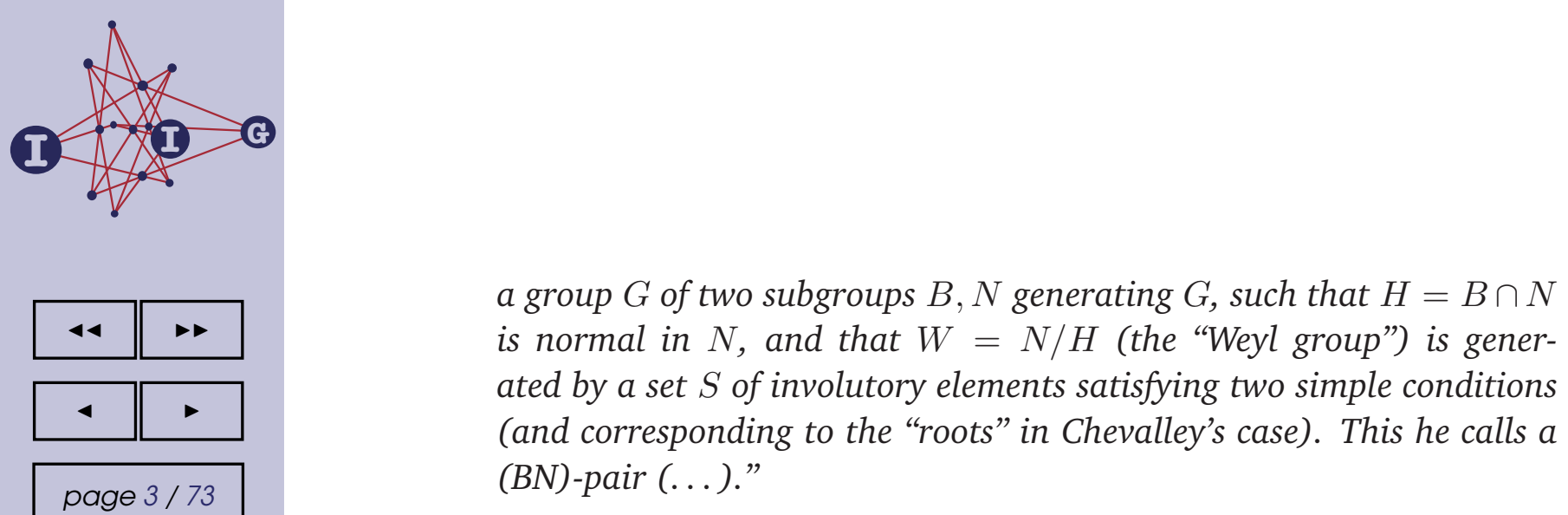

a group $G$ of two subgroups $B, N$ generating $G$, such that $H=B \cap N$ is normal in $N$, and that $W=N / H$ (the "Weyl group") is generated by a set $S$ of involutory elements satisfying two simple conditions (BN)-pair (...)."

This notion of a BN-pair was inspired to J. Tits by the decompositions in double cosets discovered by F. Bruhat [16], which had then been extended and extensively used by C. Chevalley. What J. Dieudonné called "purely grouptheoretical" in his review turned out to be the group-theoretic side of a unified geometrical approach to the whole theory, that J. Tits developed by creating the notion of buildings [14, IV §2 Exercice 15]. Exploiting beautifully the combinatorial and geometrical aspects of these objects, J. Tits was able to classify completely the irreducible buildings of rank $\geqslant 3$ with finite Weyl group [85]. A key property of these buildings is that they happen to be all highly symmetric: they enjoy the so-called Moufang property. J. Tits' classification shows furthermore that they are all related to simple algebraic groups or to classical groups in some way. J. Tits also shows that a generalization of the fundamental theorem of projective geometry holds for buildings (seen as incidence structures). This result was used by G.D. Mostow to prove his famous strong rigidity theorem for finite volume locally symmetric spaces of rank $\geqslant 2$ [62]; in this way the combinatorial aspects of Lie structures found a beautiful, deep and surprising application to differential geometry.

A few decades later, jointly with R. Weiss, J. Tits completed the extension of this classification to all irreducible Moufang buildings of rank $\geqslant 2$ with a finite Weyl group [97]. This result, combined with [12], yields a classification of all groups with an irreducible split BN-pair of rank $\geqslant 2$ with finite Weyl group. The condition that the BN-pair splits is the group-theoretic translation of the Moufang property (and has nothing to do with splitness in the sense of algebraic groups). Thus, every irreducible BN-pair of rank $\geqslant 3$ with a finite Weyl group splits. Concerning BN-pairs with finite Weyl groups, we finally note that what this group combinatorics does not cover in the theory of algebraic semisimple groups is the case of anisotropic groups. The structure of these groups is still mysterious and for more information about this, we refer to [86], [56, VIII.2.17] and [66].

A remarkable feature of the abstract notion of a BN-pair is that it does not require the Weyl group to be finite, even though J. Tits originally used them to study groups with a finite Weyl group in [83] (the BN-pairs in these groups had been constructed in his joint work with A. Borel [11]). The possibility for the Weyl group to be infinite was called to play a crucial role in another 


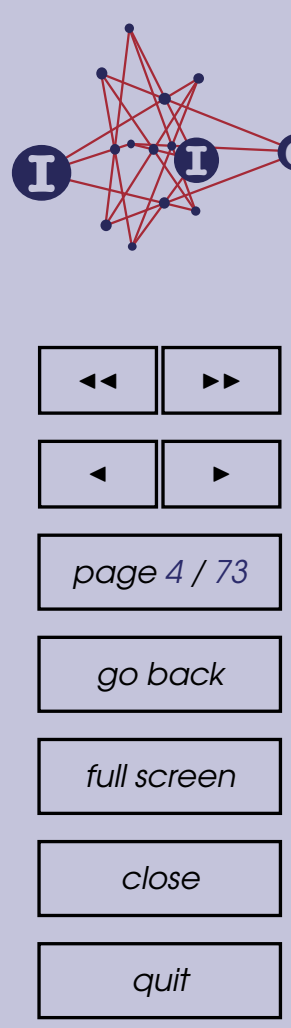

breakthrough, initiated by the discovery of affine BN-pairs in $p$-adic semisimple groups by N. Iwahori and H. Mastumoto [49]. This was taken up by F. Bruhat and J. Tits in their celebrated theory of reductive groups over local fields [17]. In the latter, a refinement of the notion of split BN-pairs was introduced, namely valuated root data (in French: données radicielles valuées). These combine the information encoded in root data with extra information on the corresponding BN-pairs coming from the valuation of the ground field. Valuated root data turned out to be classifying data for Bruhat-Tits buildings, namely the buildings constructed from the aforementioned affine BN-pairs [90].

We note that in the case of Bruhat-Tits theory, the BN-pair structure (in fact the refined structure of valuated root datum) was not a way to encode a posteriori some previously known structure results proved by algebraic group tools (as in the case of Borel-Tits theory with spherical BN-pairs and buildings). Indeed, the structure of valuated root datum, and its counterpart: the geometry of Euclidean buildings, is both the main tool and the goal of the structure theory. The existence of a valuated root datum structure on the group of rational points is proved by a very hard two-step descent argument, whose starting point is a split group. The argument involves both (singular) non-positive curvature arguments and the use of integral structures for the algebraic group under consideration. The final outcome can be nicely summed by the fact that the Bruhat-Tits building of the valuated root datum for the rational points is often the fixed point set of the natural Galois action in the building of the split group [18]. In fact, F. Bruhat and J. Tits formulate their results at such a level of generality (in particular with fields endowed with a possibly dense or even surjective valuation) that the structure of valuated root datum still makes sense while that of BN-pair doesn't in general (when the valuation is not discrete). At last, this study became complete after J. Tits' classification of affine buildings, regardless of any group action a priori [90]; roughly speaking, this classification reduces to the previous classification of spherical buildings after considering a suitably defined building at infinity. We refer to [101] for a detailed exposition of the classification in the discrete case.

At about the same time as Bruhat-Tits theory was developed, the first examples of groups with BN-pairs with infinite but non-affine Weyl groups were constructed by R. Moody and K. Teo [61] in the realm of Kac-Moody theory. The latter theory had been initiated by R. Moody and V. Kac independently a few years before in the context of classifying simple Lie algebras with growth conditions with respect to a grading. The corresponding groups (which were not so easily constructed) became known as Kac-Moody groups and were regarded as infinite-dimensional versions of the semisimple complex Lie groups. Several works in the 1980's, notably by V. Kac and D. Peterson, highlighted in- 



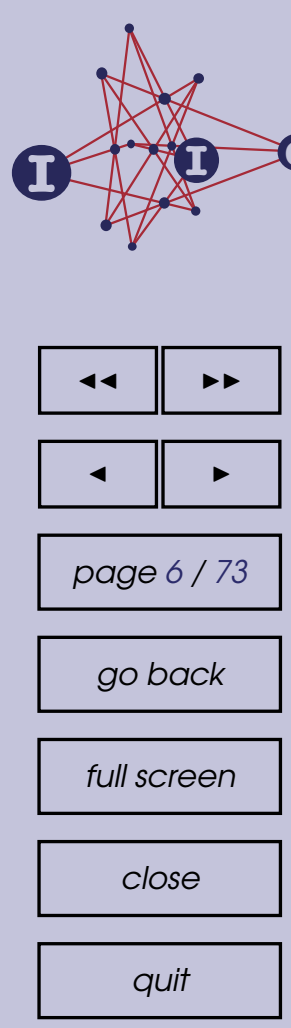

which in turn yield a pair of buildings acted upon by the ambient group $G$; this interplay between buildings and BN-pairs is then further described.

Part II: group actions on buildings and associated structure results. The second part is devoted to the algebraic results that can be derived from the existence of a sufficiently transitive group action on a building. In Section 5, we first introduce a very important tool designed by J. Tits, namely the combinatorial analogue of techniques from algebraic topology for partially ordered sets; this is very useful for some amalgamation and intersection results. Subsequently we deduce a number of basic results on the structure of groups endowed with a root group datum. In Section 6, we explain that since the automorphism group of any building carries a canonical topology, these buildings may be used to endow $G$ (admitting a root group datum) with two distinguished group topologies, with respect to which one may take metric completions; these yield two larger groups $G_{+}$and $G_{-}$containing both $G$ as a dense subgroup, and the diagonal embedding of $G$ makes it a discrete subgroup in $G_{+} \times G_{-}$. In Section 7, some simplicity results for $G_{ \pm}$and $G$ are discussed. In Section 8 we show that, under some conditions, the group $G$ admits certain nice presentations which can be used to describe classification results for root group data.

\section{Notation}

If $G$ is a group, the order of an element $g \in G$ is denoted by $o(g)$. If moreover $H$ is a subgroup of $G$, then ${ }^{g} H$ denotes the conjugate $g H^{-1}$.

\section{What this article does not cover}

The main aim of these notes is to highlight some algebraic properties common to all groups with a root group datum, with a special emphasis in those with an infinite Weyl group. However, root group data were initially designed to describe and study the combinatorial structure of rational points of isotropic simple algebraic groups, and it is far beyond the scope of this paper to describe the theory of algebraic groups. For a recent account of advanced problems in that area, we refer to [43]. Another excellent reference on root group data with finite Weyl groups is the comprehensive book by J. Tits and R. Weiss [97], which is targeted at the classification in the rank two case. The case of rank one root group data, i.e. Moufang sets, is a subject in its own right: see [33] in the same volume. 


\section{Acknowledgements}

These notes are based on a series of lectures the first author gave in the Advanced Class in Algebra at the University of Oxford during Hilary Term 2007. Thanks are due to Dan Segal for proposing him to expose this topic in the class, and to all attendants for their questions and interest. A first draft of these notes was written and circulated at the time. These were then taken over and revised when both authors jointly gave a mini-course on root group data at the conference Buildings \& Groups in Ghent in May 2007; they are grateful to the organizers of that conference for the invitation to participate actively to the event. The second author thanks É. Ghys for a useful conversation, in particular suggesting to investigate J. Tits' earliest works.

\section{Contents}

I Survey of the theory and examples 10

1 Root data 10

1.1 Root bases . . . . . . . . . . . . . . . . 10

1.1 .1 Axioms of a root basis ............ 10

1.1 .2 Products and irreducibility . . . . . . . . . . . . . 12

1.1.3 Example: the standard root basis of a Coxeter system . . . 12

1.1.4 The Weyl group is a Coxeter group . . . . . . . . . . . 13

1.1 .5 The set $\Phi(\mathbf{B})_{w} \ldots \ldots \ldots$. . . . . . . . . . . 13

1.1.6 Reflections and root subbases ............. 14

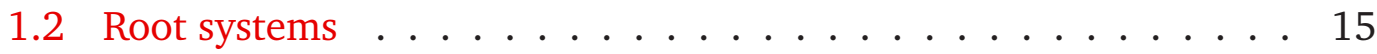

1.2.1 Root systems with respect to a root basis . . . . . . . . 15

1.2 .2 Prenilpotent sets of roots . . . . . . . . . 16

1.3 Root data . . . . . . . . . . . . . . . . 17

2 Root group data $\quad 17$

2.1 Axioms of a root group datum . . . . . . . . . . . 17

2.2 Comments on the axioms of a root group datum . . . . . . . . . 18

2.3 Root group data for root subsystems . . . . . . . . . . . . 19

2.4 A reduction ...................... 20 
6 Group topology 46

6.1 Topological completions . . . . . . . . . . . . . . 46

6.2 Levi decompositions . . . . . . . . . . . . . . . . 50

6.3 The group $\widehat{U}_{+}$and other projective limits . . . . . . . . . . . 51

6.4 Lattices . . . . . . . . . . . . . . . . . 53

7 Simplicity results $\quad \mathbf{5 5}$

full screen

7.1 Tits' transitivity lemma . . . . . . . . . . . . . . . . . 55

7.2 Topological simplicity of topological completions . . . . . . . 56

7.3 Abstract simplicity of topological completions . . . . . . . . . . . 57

7.4 Weyl transitivity of normal subgroups . . . . . . . . . . . . 59

7.5 Simplicity of lattices . . . . . . . . . . . . . . . 60

8 Curtis-Tits type presentations and existence results 61

8.1 Curtis-Tits and Steinberg type presentations of the universal central extension

8.2 Existence and classification results . . . . . . . . . . 63 


\section{Part I}

\section{Survey of the theory and examples}

\section{Root data}

go back

full screen

close

quit

ACADEMIA

PRESS

NWII

$\widehat{\underline{\text { IIIIII }}}$

UNIVERSITEIT

GENT

Root data were first introduced by M. Demazure [36, Exp. XXI] as data which classify, up to isomorphism, reductive group schemes over $\mathbb{Z}$ or split reductive algebraic groups over a given field [79, Chapters 9-10]. Demazure's original definition can be viewed as a refinement of the notion of finite root systems, taking into account the possibility to have a non-trivial (connected) central torus. However, root systems encountered in Kac-Moody theory are mostly infinite, hence the definition of a root datum we give is not Demazure's (although it is closely related). The way towards a general theory of infinite root systems has been paved by R. Moody and A. Pianzola [59] (see also [60, Chapter 5] for a more comprehensive and self-contained treatment). However, this approach has two drawbacks that we want to avoid: it implicitly excludes non-reduced root systems and it requires a certain integrality condition. The axioms we propose here follow rather closely J.-Y. Hée's approach developed in [46] (for a further comment on the comparison between these references, see Remark 1.1.1 below). We note that in another vein of generalization, N. Bardy has developed an abstract theory of root systems covering R. Borcherds' work using Lie algebras for number theory [7]; this topic will not be covered here.

The content of this section is very simple: we first define root bases, which are designed to generate root systems, which themselves are the index sets of the combinatorics of root group data.

\subsection{Root bases}

\subsubsection{Axioms of a root basis}

Let $V$ be a real vector space. A root basis for $V$ is a pair $\mathbf{B}=\left(\Pi, \Pi^{\vee}=\left\{\alpha^{\vee}\right\}_{\alpha \in \Pi}\right)$ where $\Pi$ is a (nonempty) subset of $V$ and $\Pi^{\vee}$ is a set consisting of an element $\alpha^{\vee} \in V^{*}$ associated to each element $\alpha \in \Pi$, submitted to the following conditions:

(RB1) For each $\alpha \in \Pi$, we have $\left\langle\alpha, \alpha^{\vee}\right\rangle=2$.

(RB2) For all $\alpha, \beta \in \Pi$ with $\alpha \neq \beta$, we have either $\left\langle\alpha, \beta^{\vee}\right\rangle=\left\langle\beta, \alpha^{\vee}\right\rangle=0$ or $\left\langle\alpha, \beta^{\vee}\right\rangle\left\langle 0,\left\langle\beta, \alpha^{\vee}\right\rangle<0\right.$ and $\left\langle\alpha, \beta^{\vee}\right\rangle\left\langle\beta, \alpha^{\vee}\right\rangle \in\left\{4 \cos ^{2}\left(\frac{\pi}{k}\right) \mid k \in \mathbb{Z}\right\} \cup \mathbb{R}_{\geqslant 4}$. 

for all $s, t \in S$. We also set $f_{s}=2\left(\cdot, e_{s}\right) \in V^{*}$ for each $s \in S$. Then $\mathbf{B}(W, S)=$ $\left(\left\{e_{s}\right\}_{s \in S},\left\{f_{s}\right\}_{s \in S}\right)$ is a free root basis. Note that (RB3) obviously holds here because the $e_{s}$ 's are linearly independent. This is called the standard root basis associated with $(W, S)$.

Remark 1.2. It is well-known that the map $W \rightarrow \mathrm{GL}(V)$ attaching to each $s \in S$ the reflection $\sigma_{s}: v \mapsto v-2\left(e_{s}, v\right) e_{s}$ is an injective group homomorphism [14, V.4].

\subsubsection{The Weyl group is a Coxeter group}

A basic result on root bases is the following:

Theorem 1.3. Let $\mathbf{B}=\left(\Pi, \Pi^{\vee}\right)$ be a root basis. We have the following:

(i) The ordered pair $(W, S)$ is a Coxeter system. Furthermore, for all distinct $\alpha, \beta \in \Pi$, the order $o\left(r_{\alpha} r_{\beta}\right)$ of $r_{\alpha} r_{\beta}$ is equal to $k$ (resp. $\infty$ ) if $A_{\alpha, \beta} A_{\beta, \alpha}=$ $4 \cos ^{2}\left(\frac{\pi}{k}\right)$ (resp. if $A_{\alpha, \beta} A_{\beta, \alpha} \geqslant 4$ ).

(ii) We have $\Phi(\mathbf{B})=\Phi(\mathbf{B})_{+} \cup \Phi(\mathbf{B})_{-}$.

Proof. The axioms (RB1)-(RB3) imply that any pair $\{\alpha, \beta\}$ of elements of $\Pi$ is linearly independent. In other words $\mathbf{B}_{\{\alpha, \beta\}}$ is a free root basis. By [46, (2.11)], it is thus a root basis in the sense of [loc. cit.]. Now the arguments of $[46,(2.10)]$ show that (ii) holds and allow moreover to apply verbatim the proof of $[14$, Ch. $5, \S 4$, Th. 1], which yields (i). Finally, the rule that computes the order of $r_{\alpha} r_{\beta}$ is established in [46, Prop. 1.23].

Remark 1.4. The result [14, Ch. 5, $\S 4$, Th. 1] quoted above is due to J. Tits; a more general version as the one in [loc. cit.] is stated in [96, Lemme 1].

\subsubsection{The set $\Phi(\mathrm{B})_{w}$}

Let $\mathbf{B}=\left(\Pi, \Pi^{\vee}=\left\{\alpha^{\vee}\right\}_{\alpha \in \Pi}\right)$ and let $W=W(\mathbf{B})$ be its Weyl group. For each $w \in W$, we set

$$
\Phi(\mathbf{B})_{w}=\left\{\alpha \in \Phi(\mathbf{B})_{+} \mid w \cdot \alpha \in \Phi(\mathbf{B})_{-}\right\} .
$$

Lemma 1.5. Let $\ell$ denote the word length in $W$ with respect to $S$, i.e., for any $w \in W$ we set: $\ell(w)=\min \left\{m \in \mathbb{N}: w=s_{1} s_{2} \cdots s_{m}\right.$ with each $s_{i}$ in $\left.S\right\}$.

(i) For all $w \in W$ and $\alpha \in \Pi$, we have

$$
\begin{aligned}
& \ell\left(r_{\alpha} w\right)>\ell(w) \text { if and only if } w^{-1} \cdot \alpha \in \Phi(\mathbf{B})_{+}, \quad \text { and } \\
& \ell\left(r_{\alpha} w\right)<\ell(w) \text { if and only if } w^{-1} \cdot \alpha \in \Phi(\mathbf{B})_{-} .
\end{aligned}
$$





\subsection{Root systems}

\subsubsection{Root systems with respect to a root basis}

Given a root basis $\mathbf{B}=\left(\Pi, \Pi^{\vee}=\left\{\alpha^{\vee}\right\}_{\alpha \in \Pi}\right)$, a B-root system is a subset $\Phi$ of $V \backslash\{0\}$ which is $W(\mathbf{B})$-invariant, contained in $\{\lambda \alpha \mid \alpha \in \Phi(\mathbf{B}), \lambda \in \mathbb{R}\}$ and such that for each $\alpha \in \Pi$, the set $\Phi \cap \mathbb{R} \alpha$ is finite and non-empty. The set

$$
\Pi_{\Phi}=\left\{\beta \in \Phi \mid \beta=\lambda \alpha \text { for some } \alpha \in \Pi \text { and } \lambda \in \mathbb{R}_{+}\right\}
$$

full screen

close quit

is called the basis of $\Phi$. The B-root system $\Phi$ is called reduced if $\Phi \cap \mathbb{R} \alpha$ has cardinality 2 for each $\alpha \in \Pi$, i.e., if $\Phi \cap \mathbb{R} \alpha=\{ \pm \alpha\}$. Given a B-root system $\Phi$, we set $\Phi_{+}=\Phi \cap \mathbb{R}_{+} \Phi(\mathbf{B})_{+}$and $\Phi_{-}=\Phi \cap \mathbb{R}_{+} \Phi(\mathbf{B})_{-}$. By Theorem 1.1.4(ii), we have $\Phi=\Phi_{+} \cup \Phi_{-}$.

Note that by Section 1.1.6, there is a reflection $r_{\beta} \in W$ associated with every root $\beta$ of a B-root system $\Phi$. A subset $\Psi$ of $\Phi$ is called a B-root subsystem if $\Psi$ is $r_{\beta}$-invariant for each $\beta \in \Psi$. Note that a root subsystem is a root system in a root subbase of $\mathbf{B}$, whose Weyl group is $W_{\Psi}$. We say that the $\mathbf{B}$-root subsystem $\Psi$ is parabolic if $W_{\Psi}$ is a parabolic subgroup of $W$, namely it is the Weyl group of a parabolic root subbasis.

Given any $\Psi \subset \Phi$, the set $\langle\Psi\rangle=\left\{w \cdot \alpha \mid \alpha \in \Psi, w \in W_{\Psi}\right\}$, where $W_{\Psi}=\left\langle r_{\beta}\right|$ $\beta \in \Psi\rangle$, is a root subsystem. It is called the root subsystem generated by $\Psi$.

For each $w \in W(\mathbf{B})$, we let

$$
\Phi_{w}=\left\{\alpha \in \Phi_{+} \mid w \cdot \alpha \in \Phi_{-}\right\} .
$$

Note that a decomposition similar to that of Lemma 1.5(iii) holds for $\Phi_{w}$. In particular, this shows that the set $\Phi_{w}$ is finite.

Lemma 1.7. Let $\mathbf{B}=\left(\Pi, \Pi^{\vee}\right)$ be a root basis. We have the following:

(i) $\Phi(\mathbf{B})$ is a reduced $\mathbf{B}$-root system if and only if for all $\alpha, \beta \in \Pi$ such that the order $o\left(r_{\alpha} r_{\beta}\right)$ is odd, one has $A_{\alpha, \beta}=A_{\beta, \alpha}$.

(ii) If there exists a B-root system, then $\Phi(\mathbf{B})$ is a $\mathbf{B}$-root system.

Proof. (i) By [46, (2.17)], the set $\Phi(\mathbf{B})$ is a reduced root system if and only if $\Phi\left(\mathbf{B}_{\{\alpha, \beta\}}\right)$ is a reduced root system for all distinct $\alpha, \beta \in \Pi$. Clearly, the subspace $V_{\alpha \beta}$ of $V$ spanned by $\alpha$ and $\beta$ is $W_{\{\alpha, \beta\}}$-invariant. Moreover, the $W_{\{\alpha, \beta\}}$-action on $V_{\alpha \beta}$ preserves the symmetric bilinear form $(\cdot, \cdot)$ defined by:

$$
(\alpha, \alpha)=-A_{\alpha, \beta}, \quad(\beta, \beta)=-A_{\beta, \alpha}, \quad(\alpha, \beta)=-\frac{A_{\alpha, \beta} A_{\beta, \alpha}}{2} .
$$





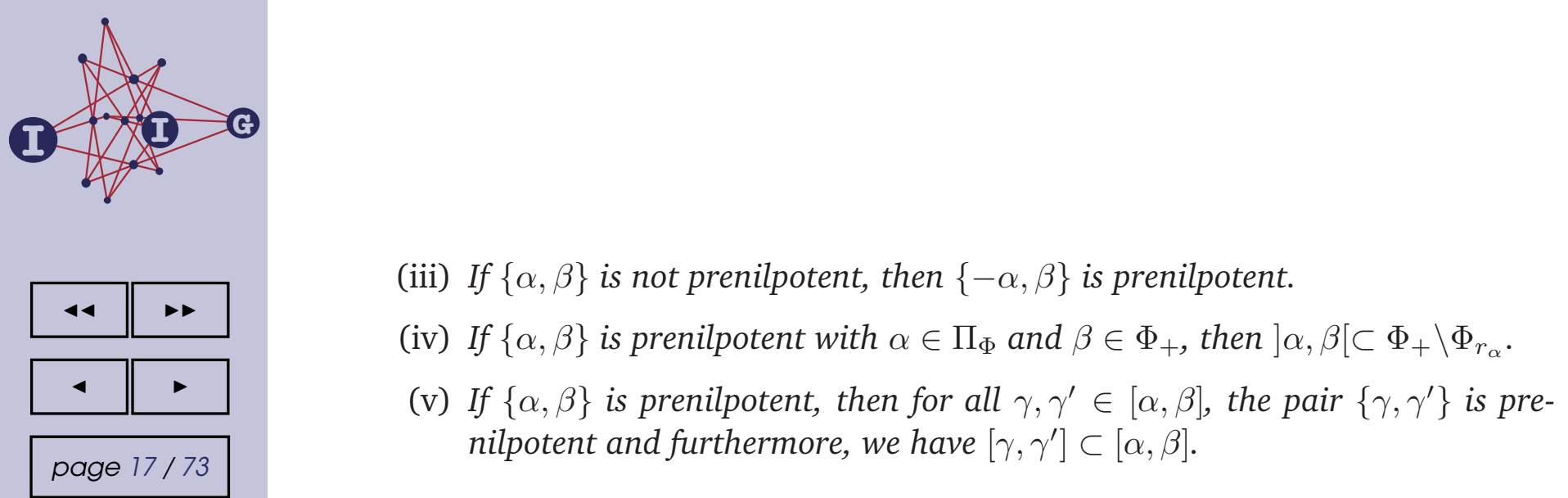

Proof. (i) By Theorem 1.1.4(i), the Weyl group of a finite root system is a finite Coxeter group. The (unique) element of maximal length maps every positive root of this system to a negative one. The desired assertion follows easily.

(ii) By [60, Ch. 5, Prop. 8], we have $\left\langle\alpha, \beta^{\vee}\right\rangle>0$ (resp. $<0$ ) if and only if $\left\langle\beta, \alpha^{\vee}\right\rangle>0$. Now if $\left\langle\alpha, \beta^{\vee}\right\rangle<0$, then the set $[\alpha, \beta]_{\text {lin }}$ is infinite since the group $\left\langle r_{\alpha}, r_{\beta}\right\rangle$ is infinite. Therefore, the pair $\{\alpha, \beta\}$ cannot be prenilpotent, since the set $[\alpha, \beta]$, which contains $[\alpha, \beta]_{\text {lin }}$, is prenilpotent, hence finite. For the converse statement, see [23, Lemma 2.3]

(iii) Follows from (i) and (ii).

(iv) Follows from Lemma 1.5(iii).

(v) We have mentioned above that a nilpotent set of roots is prenilpotent. Moreover, it is clear from the definition that any subset of a prenilpotent set of roots is prenilpotent. Thus $\left\{\gamma, \gamma^{\prime}\right\}$ is prenilpotent. The inclusion $\left[\gamma, \gamma^{\prime}\right] \subset[\alpha, \beta]$ follows from the definitions.

\subsection{Root data}

A root datum consists in a root basis $\mathbf{B}=\left(\Pi, \Pi^{\vee}\right)$ such that $A_{\alpha, \beta}=A_{\beta, \alpha}$ for all $\alpha, \beta \in \Pi$ such that $o\left(r_{\alpha} r_{\beta}\right)$ is finite and odd, together with a B-root system $\Phi$. All the vocabulary used to qualify root bases (e.g. free, integral, irreducible, etc.) will be used for root data as well, according as the property in question holds for the underlying root basis.

\section{Root group data}

\subsection{Axioms of a root group datum}

We are now ready to introduce the main object of study. Let $G$ be a group and $E=(\mathbf{B}, \Phi)$ be a root datum. Thus $\mathbf{B}=\left(\Pi, \Pi^{\vee}\right)$ is a root basis in a real vector space $V$ which will be held fixed throughout, and $\Phi$ is a $\mathbf{B}$-root system. 
A root group datum of type $E$ for $G$ (formerly called a twin root datum) is a tuple $\left\{U_{\alpha}\right\}_{\alpha \in \Phi}$ of subgroups of $G$ which, setting

$$
U_{+}=\left\langle U_{\alpha} \mid \alpha \in \Phi_{+}\right\rangle \quad \text { and } \quad U_{-}=\left\langle U_{\alpha} \mid \alpha \in \Phi_{-}\right\rangle
$$

satisfies the following axioms.

(RGD0) For all $\alpha \in \Phi$, we have $U_{\alpha} \neq\{1\}$ and moreover $G=\left\langle U_{\alpha} \mid \alpha \in \Phi\right\rangle$.

(RGD1) For each $\beta \in \Pi_{\Phi}$, we have $U_{\beta} \not \subset U_{-}$.

(RGD2) For each $\beta \in \Pi_{\Phi}$ and each $u \in U_{\beta} \backslash\{1\}$, there exists an element $\mu(u) \in$ $U_{-\beta} . u . U_{-\beta}$ such that $\mu(u) U_{\alpha} \mu(u)^{-1}=U_{r_{\beta} . \alpha}$ for all $\alpha \in \Phi$.

(RGD3) For each prenilpotent pair $\{\alpha, \beta\} \subset \Phi$, we have

$$
\left.\left[U_{\alpha}, U_{\beta}\right] \subset\left\langle U_{\gamma}\right| \gamma \in\right] \alpha, \beta[\rangle
$$

(RGD4) For each $\beta \in \Pi_{\Phi}$ there exists $\beta^{\prime} \in \Phi_{r_{\beta}}$ such that $U_{\alpha} \subset U_{\beta^{\prime}}$ for each $\alpha \in \Phi_{r_{\beta}}$.

The subgroups $U_{\alpha}$ of $G$ are called root subgroups.

\subsection{Comments on the axioms of a root group datum}

Remarks 2.1. (1) Combining (RGD0) with (RGD2), it follows that $G$ is generated by the set

$$
\left\{U_{\beta} \mid \beta \in \Pi_{\Phi}\right\} \cup\left\{U_{-\beta} \mid \beta \in \Pi_{\Phi}\right\}
$$

(2) As it is the case for root bases, one obtains new systems of root subgroups from existing ones by taking products. We leave it to the reader to perform these constructions in details. In particular, if the root datum $E$ is not irreducible, then $G$ is a commuting product of subgroups, each one endowed with a root group datum indexed by a root subsystem of $E$.

(3) We will establish in Corollary 5.3(iii) below that $U_{-\beta} \not \subset U_{+}$for each $\beta \in \Pi_{\Phi}$. Thus, the whole theory is 'symmetric in + and -', although (RGD1) seems to break the symmetry at a first glance. In other words, if $\left\{U_{\alpha}\right\}_{\alpha \in \Phi}$ is a root group datum for $G$, then so is $\left\{U_{-\alpha}\right\}_{\alpha \in \Phi}$.

(4) A strengthened version of axiom (RGD3) is the following: 


\subsection{A reduction}

Let $E=(\mathbf{B}, \Phi)$ be a root datum. By definition of root data, Lemma 1.2.1(i) shows that $\Phi(\mathbf{B})$ is a reduced $\mathbf{B}$-root system. For each $\alpha \in \Phi(\mathbf{B})$, we set

$$
\left.U_{(\alpha)}=\left\langle U_{\beta}\right| \beta \in \Phi, \beta=\lambda \alpha \text { for some } \lambda>0\right\rangle .
$$

Lemma 2.4. The system $\left\{U_{(\alpha)}\right\}_{\alpha \in \Phi(\mathbf{B})}$ is a root group datum of type $(\mathbf{B}, \Phi(\mathbf{B}))$ for $G$.

full screen

close

quit

Proof. It is clear from the definition that (RGD0) and (RGD1) hold. By (RGD4) for the original root group datum, we deduce that for each $\alpha \in \Pi$ there exists $\beta \in \Pi_{\Phi}$ such that $U_{(\alpha)}=U_{\beta}$. Therefore (RGD2) holds as well. The fact that (RGD3) holds follows easily by combining (RGD3) for the original root group datum with Lemma 1.2.2(v). Finally, since $\Phi(\mathbf{B})$ is reduced, the axiom (RGD4) is clearly satisfied.

The lemma shows that any root group datum for a group $G$ yields a root group datum for $G$ indexed by a reduced root system. Most structure results on groups endowed with a root group datum assume that the underlying root system is reduced. In view of the reduction presented above, this assumption causes no loss of generality.

\subsection{Example: rank one groups}

The purpose of the present subsection and the following ones is to describe a first set of examples of groups admitting a root group datum.

A group $G$ is called a rank one group if it admits a root group datum indexed by a root system of rank one, which can be assumed to be reduced in view of Section 2.4. Equivalently $G$ possesses nontrivial subgroups $U_{+}$and $U_{-}$, whose union generates $G$ and such that for each $u \in U_{+} \backslash\{1\}$ there exists $\mu(u) \in$ $U_{-} . u . U_{-}$such that conjugation by $\mu(u)$ swaps $U_{+}$and $U_{-}$. It is easy to see that the latter condition is equivalent to the following, where $A=U_{+}$and $B=U_{-}$:

$$
\text { for each } a \in A \backslash\{1\} \text {, there exists } b \in B \text { such that }{ }^{b} A={ }^{a} B \text {. }
$$

For instance, the group $G=\mathrm{SL}_{2}(k)$, where $k$ is any field, is a rank one group with root subgroups

$$
A=\left\{\left(\begin{array}{ll}
1 & x \\
0 & 1
\end{array}\right) \mid x \in k\right\} \quad \text { and } \quad B=\left\{\left(\begin{array}{ll}
1 & 0 \\
x & 1
\end{array}\right) \mid x \in k\right\} .
$$

Indeed, given $a=\left(\begin{array}{ll}1 & x \\ 0 & 1\end{array}\right)$ with $x \in k^{\times}$, one has ${ }^{b} A={ }^{a} B$ with $b=\left(\begin{array}{cc}1 & 0 \\ -x^{-1} & 1\end{array}\right)$. 




Finally, we remark that, in view of the root space decomposition of $\mathfrak{g}(A)$, the subalgebra $\mathfrak{x}_{i}$ generated by $e_{i}$ and $f_{i}$ is 3-dimensional. Now, if $a_{i i}=0$, then $\mathfrak{x}_{i}$ is isomorphic to a Heisenberg Lie algebra. If $a_{i i} \neq 0$, then $\mathfrak{x}_{i}$ is not solvable and, hence, it must be isomorphic to $\mathfrak{s l}_{2}(\mathbb{C})$.

\subsection{Kac-Moody algebras}

\subsubsection{The root basis and its canonical root system}

The Lie algebra $\mathfrak{g}(A)$ is called a Kac-Moody algebra if the matrix $A$ is a generalized Cartan matrix, namely if $A \in \mathbb{Z}^{n \times n}$ and moreover $a_{i i}=2, a_{i j} \leqslant 0$ and $a_{i j}=0 \Leftrightarrow a_{j i}=0$ for all $i \neq j \in\{1, \ldots, n\}$. This is equivalent to the requirement that $\mathbf{B}(A)=\left(\Pi, \Pi^{\vee}\right)$ be an integral root basis. Note that $\mathbf{B}(A)$ is free by assumption. Let $S=S(\mathbf{B}(A))$ and $W=W(\mathbf{B}(A))$ be the Weyl group of $\mathbf{B}(A)$. By Theorem 1.1.4(i), for all distinct $\alpha, \beta \in \Pi$ we have $o\left(r_{\alpha} r_{\beta}\right)=2,3,4,6$ or $\infty$ according as $A_{\alpha, \beta} A_{\beta, \alpha}=0,1,2,3$ or $\geqslant 4$. In particular the set $\Phi(\mathbf{B}(A))$ is a reduced root system by Lemma 1.2.1(i). We will see in the next subsection that that the root system $\Phi(\mathbf{B}(A))$ has in fact a Lie-theoretic interpretation in the present context.

\subsection{2. $\quad$ Lifting the Weyl group}

A basic fact on Kac-Moody algebras is that they satisfy Serre's relations:

$$
\left(\operatorname{ad} e_{i}\right)^{1-a_{i j}} e_{j}=0 \quad \text { and } \quad\left(\operatorname{ad} f_{i}\right)^{1-a_{i j}} f_{j}=0
$$

for all $i \neq j$. This follows from basic computations in $\mathfrak{s l}_{2}(\mathbb{C})$-modules, see [50, $\S 3.3]$. An immediate consequence is the following:

Lemma 3.3. The operators ad $e_{i}$ and ad $f_{i}$ are locally nilpotent on $\mathfrak{g}(A)$ for all $i=1, \ldots, n$.

Proof. Recall that a linear operator $A \in \operatorname{End}(V)$ of a vector space $V$ is called locally nilpotent if every vector $v \in V$ is contained in a finite-dimensional $A$-stable subspace $U$ such that the restriction of $A$ to $U$ is nilpotent. In view of the definition of $\tilde{\mathfrak{g}}(A)$ and $\mathfrak{g}(A)$, we have $\left(\operatorname{ad} e_{i}\right)^{2} h=0$ for any $h \in \mathfrak{h}$. In view of Serre's relations, it follows that for any generator $x$ of the Lie algebra $\mathfrak{g}(A)$ there is an integer $N_{x}$ such that $\left(\operatorname{ad} e_{i}\right)^{N_{x}} x=0$. Now, using Leibniz' rule (note that ad $e_{i}$ is a derivation of $\mathfrak{g}(A)$ by Jacobi's identity), one deduces by a straightforward induction on iterated commutators of the generators of $\mathfrak{g}(A)$ that $\operatorname{ad} e_{i}$ is locally nilpotent. Similar discussions apply to ad $f_{i}$. 
From the lemma it follows that

$$
\exp \operatorname{ad} e_{i}=\sum_{m=0}^{\infty} \frac{1}{m !}\left(\operatorname{ad} e_{i}\right)^{m}
$$

is a well-defined automorphism of $\mathfrak{g}(A)$.

Now, for each $i \in\{1, \ldots, n\}$, we consider the automorphism

$$
r_{i}=\exp \operatorname{ad} e_{i} \cdot \exp \operatorname{ad}-f_{i} \cdot \exp \operatorname{ad} e_{i} \in \operatorname{Aut}(\mathfrak{g}(A)) .
$$

Note that $r_{i}$ stabilizes the subalgebra $\mathfrak{x}_{i} \simeq \mathfrak{s l}_{2}(\mathbb{C})$ and acts on it as the involution $e_{i} \mapsto-f_{i}, f_{i} \mapsto-e_{i}, \alpha_{i}^{\vee} \mapsto-\alpha_{i}^{\vee}$. Furthermore, straightforward computations show that

$$
r_{i}(h)=h-\left\langle\alpha_{i}, h\right\rangle \alpha_{i}^{\vee}
$$

for all $h \in \mathfrak{h}$. In particular, the automorphism $r_{i}$ preserves $\mathfrak{h}$ and, consequently, preserves the corresponding root space decomposition of $\mathfrak{g}(A)$. In other words, $r_{i}$ induces a permutation of $\Phi$, which we denote by $r_{i}^{\vee}$. In fact, one can easily compute the action of $r_{i}^{\vee}$ on $\Phi$ by transforming the equation $[h, x]=\langle\alpha, h\rangle . x$ (satisfied by all $h \in \mathfrak{h}, x \in \mathfrak{g}_{\alpha}$ and $\alpha \in \Phi$ ) by $r_{i}$. Routine computations then show that

$$
r_{i}^{\vee}(\alpha)=\alpha-\left\langle\alpha, \alpha_{i}^{\vee}\right\rangle \cdot \alpha_{i} .
$$

This extends to a linear action of $r_{i}^{\vee}$ on $\mathfrak{h}^{*}$ which is nothing but the dual action of $r_{i}$. The following result sums up the preceding discussion:

Proposition 3.4. The canonical $\mathbf{B}(A)$-root system $\Phi(\mathbf{B}(A))$ identifies in a canonical way to a subset ${ }^{\text {re }} \Phi$ of the set of roots $\Phi$ of the Lie algebra $\mathfrak{g}(A)$.

Note that we recover the fact that $\Phi(\mathbf{B}(A))$ is reduced thanks to Equation (3.3).

A remarkable feature of Kac-Moody theory is that $\Phi$ is real, i.e. $\Phi={ }^{r e} \Phi$, if and only if $\mathfrak{g}(A)$ is finite-dimensional, in which case it is a well understood semisimple Lie algebra, see [50, Th. 5.6]. The elements of ${ }^{i m} \Phi=\Phi \backslash{ }^{\text {re }} \Phi$ are called imaginary roots.

An important open problem of the theory is to compute the dimension of the root space $\mathfrak{g}_{\alpha}$ for $\alpha$ imaginary; recall that Equation (3.2) provides a rough upper-bound. In view of (3.3), we have $\operatorname{dim} \mathfrak{g}_{\alpha}=1$ for any $\alpha \in{ }^{\text {re }} \Phi$. For such a root $\alpha \in{ }^{\mathrm{re}} \Phi$, we set

$$
U_{\alpha}=\left\langle\exp \operatorname{ad} x \mid x \in \mathfrak{g}_{\alpha}\right\rangle
$$

which is a well-defined one-parameter subgroup of $\operatorname{Aut}(\mathfrak{g}(A))$ since ad $x$ is locally nilpotent by the lemma above. 
Note also that for each $i$, the reflection $r_{\alpha_{i}}$ of the root basis $\mathbf{B}(A)$ coincides with the restriction of $r_{i}^{\vee}$ to the $\mathbb{R}$-form $\mathfrak{h}_{\mathbb{R}}^{*}$ of $\mathfrak{h}^{*}$. The Weyl group $W<\mathrm{GL}\left(\mathfrak{h}_{\mathbb{R}}^{*}\right)$ is thus isomorphic to the subgroup of $G L(\mathfrak{h})$ (resp. $G L\left(\mathfrak{h}^{*}\right)$ ) generated by the corresponding restrictions of the $r_{i}$ 's (resp. $r_{i}^{\vee}$ ). Note however that the subgroup of $\operatorname{Aut}(\mathfrak{g}(A))$ generated by the $r_{i}$ 's is not isomorphic to $W$, but to an extension of $W$ by an elementary abelian 2-group of rank $n$. This extended Weyl group is studied by J. Tits in [84].

\subsection{Root group data for Kac-Moody groups}

Maintain the notation of the previous subsection. We let moreover $G$ be the subgroup of $\operatorname{Aut}(\mathfrak{g}(A))$ generated by the $U_{\alpha}$ 's. The group $G$ is called the adjoint Kac-Moody group of type $A$ over $\mathbb{C}$.

Theorem 3.5. The tuple $\left\{U_{\alpha}\right\}_{\alpha \in \mathrm{re}_{\Phi}}$ is a root group datum for $G$, satisfying also (RGD3) lin.

Proof. Condition (RGD0) holds by construction. For (RGD1), note that $U_{+}$stabilizes the subalgebra $\mathfrak{n}_{+}$generated by the $e_{i}$ 's. Moreover, the group $U_{-\alpha_{i}}$ stabilizes the subalgebra $\mathfrak{x}_{i}$. It follows that $U_{-\alpha_{i}} \not \subset U_{+}$, otherwise $U_{-\alpha_{i}}$ would stabilize $\mathfrak{x}_{i} \cap \mathfrak{n}_{+}=\mathfrak{g}_{\alpha_{i}}$, which is absurd. A similar argument shows that $U_{\alpha_{i}} \not \subset U_{-}$, hence (RGD1) holds. Condition (RGD2) is satisfied as follows from the preceding discussion on the automorphisms $r_{i} \in \operatorname{Aut}(\mathfrak{g}(A))$. Moreover (RGD4)

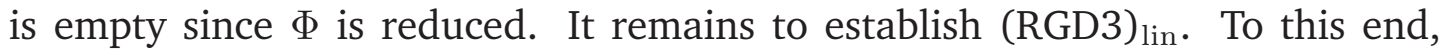
for any prenilpotent pair $\{\alpha, \beta\} \subset{ }^{\text {re }} \Phi$ we let $\mathfrak{g}_{[\alpha, \beta]}$ be the vector space generated by all root spaces $\mathfrak{g}_{\gamma}$ with $\gamma \in[\alpha, \beta]_{\text {lin }}=\Phi \cap\left(\mathbb{R}_{+} \alpha+\mathbb{R}_{+} \beta\right)$. Thus we have $\mathfrak{g}_{[\alpha, \beta]}=\bigoplus_{\gamma \in[\alpha, \beta]_{\text {lin }}} \mathfrak{g}_{\gamma}$ and $\mathfrak{g}_{[\alpha, \beta]}$ is finite-dimensional since nilpotent sets of roots are necessarily finite by Section 1.2.2. Moreover, the rule (3.1) shows that $\mathfrak{g}_{[\alpha, \beta]}$ is in fact a nilpotent subalgebra.

Let now $\widetilde{U}_{[\alpha, \beta]}$ be the simply connected complex Lie group with Lie algebra $\mathfrak{g}_{[\alpha, \beta]}$. Thus $\widetilde{U}_{[\alpha, \beta]}$ is nothing but the set $\mathfrak{g}_{[\alpha, \beta]}$ endowed with a composition law $(u, v) \mapsto u * v$ given by the Baker-Campbell-Hausdorff formula. We also denote by $U_{[\alpha, \beta]}$ the subgroup of $\operatorname{Aut}(\mathfrak{g}(A))$ generated by exp ad $x$ for $x \in \mathfrak{g}_{[\alpha, \beta]}$. Now, it follows from the definitions that there is a canonical homomorphism

$$
\varphi: \widetilde{U}_{[\alpha, \beta]} \rightarrow U_{[\alpha, \beta]} \cdot
$$

Furthermore, denoting by $\widetilde{U}_{\gamma}$ the one-parameter subgroup of $\widetilde{U}_{[\alpha, \beta]}$ with Lie algebra $\mathfrak{g}_{\gamma}$ for each $\gamma \in[\alpha, \beta]$, we have $\varphi\left(\widetilde{U}_{\gamma}\right)=U_{\gamma}$ and we obtain a product decomposition

$$
\widetilde{U}_{[\alpha, \beta]}=\prod_{\gamma \in[\alpha, \beta]_{l i n}} \widetilde{U}_{\gamma}
$$



It follows from the axioms that the group $W$ is a Coxeter group and that $(W, S)$ is a Coxeter system [14, Ch. IV, $\S 2$, Th. 2]. Another important consequence is the following decomposition of $G$, called Bruhat decomposition [14, Ch. IV, $\S 2$, Th. 1]:

$$
G=\bigsqcup_{w \in W} B w B
$$

In other words, the double cosets of $B$ in $G$ are in one-to-one correspondence with the elements of $W$.

An important concept associated with BN-pairs is that of a parabolic subgroup. Given any subset $J \subset S$, it follows from the axiom (BN3) that the set $P_{J}=\bigsqcup_{w \in W_{J}} B w B$ is a subgroup of $G$ containing $B$, which is called a standard parabolic subgroup of type $J$. In fact, it follows from the Bruhat decomposition that any subgroup of $G$ containing $B$ is obtained in this way [14, Ch. IV, $\S 2$, Th. 3].

\subsubsection{BN-pairs from root group data}

As before, let now $\mathbf{B}=\left(\Pi, \Pi^{\vee}\right)$ be a root basis and $E=(\mathbf{B}, \Phi)$ be a root datum. Let also $G$ be a group endowed with a root group datum $\left\{U_{\alpha}\right\}_{\alpha \in \Phi}$ of type $E$. We will also assume in this subsection that $\Phi=\Phi(\mathbf{B})$ is the canonical root system of $\mathbf{B}$; in particular it is reduced. This assumption causes no loss of generality in view of Lemma 2.4.

In order to construct BN-pairs for $G$, we introduce the following additional notation:

$$
\begin{aligned}
& T=\left\langle\mu(u) \mu(v) \mid u, v \in U_{\alpha} \backslash\{1\}, \alpha \in \Pi\right\rangle, \\
& N=\left\langle\mu(u) \mid u \in U_{\alpha} \backslash\{1\}, \alpha \in \Pi\right\rangle . T
\end{aligned}
$$

and

$$
B_{ \pm}=T \cdot U_{ \pm}
$$

Clearly $T$ normalizes each root group $U_{\alpha}$, in particular $B_{+}$and $B_{-}$are subgroups of $G$ and we have $U_{ \pm} \triangleleft B_{ \pm}$. Given $\alpha \in \Pi$ and $u \in U_{\alpha} \backslash\{1\}$, we denote by $r_{\alpha}$ the coset $\mu(u) . T \subset N / T$. Note that this is indeed independent of the choice of $u \in U_{\alpha} \backslash\{1\}$. Finally we set

$$
S=\left\{r_{\alpha} \mid \alpha \in \Pi\right\}
$$

The expected relation between root group data and BN-pairs is the following statement: 
Theorem 4.1. The tuple $\left(B_{ \pm}, N, S\right)$ is a BN-pair for $G$.

The proof of this theorem is surprisingly difficult. The tools are elementary, but the proof sketched by J. Tits is a very clever and fairly indirect one. The full details, which are involved and quite technical, are given for the first time in the proof of Theorem 8.80 in [3]. The hardest point is to prove that for a root group datum as above, we have: $B_{+} \cap U_{-}=\{1\}$. For this (and for other purposes among which are amalgamation theorems), J. Tits introduced a combinatorial theory of coverings of partially ordered sets [89], which we sketch very briefly in 5.1. For a careful analysis of the proof, we recommend $[3,8.6]$, which in fact contains the first detailed written treatment of this proof; see also [71, §3] for a less detailed version, following a suggestion from [1].

Corollary 4.2. We have $B_{-}=N_{G}\left(U_{-}\right)$.

Proof. Since $U_{-}$is normal in $B_{-}$by definition, we have $B_{-} \subset N_{G}\left(U_{-}\right)$. In view of the theorem, this implies that $N_{G}\left(U_{-}\right)=P_{J}^{-}$for some $J \subset S$ since every subgroup containing $B_{-}$is a parabolic subgroup. Now if $J \neq \varnothing$, then $r_{\alpha} \in J$ for some $\alpha \in \Pi$ and hence $U_{\alpha} \in P_{J}^{-}=N_{G}\left(U_{-}\right)$. But we have just seen in the proof of (BN4) that $U_{\alpha} \not \subset N_{G}\left(U_{-}\right)$. Thus $J=\varnothing$ and $N_{G}\left(U_{-}\right)=B_{-}$.

\subsection{Coset geometries}

The purpose of the next sections is to show that a group $G$ endowed with a root group datum possesses two natural actions on two distinguished buildings, which are associated to $G$ via BN-pairs constructed from the root group datum. Actions on buildings are very helpful in exploring the structure of the groups acting, as it will become clear in the subsequent study of $G$.

As we will see below, the construction of the building associated to a group with a BN-pair is a special example of a coset geometry associated to a group endowed with an inductive system of subgroups, and it is appropriate to start by defining the latter concept.

The coset geometry is obtained by the following construction. Let $G$ be a group and let $\left\{G_{a}\right\}_{a \in F}$ be a system of subgroups indexed by some set $F$ (in such a way that $G_{a} \neq G_{b}$ for $a \neq b$ ). The index set $F$ is partially ordered by the inclusion of subgroups:

$$
a \leqslant b \Longleftrightarrow G_{a} \subset G_{b} .
$$

We view $\left\{G_{a}\right\}_{a \in F}$ as an inductive system, all of whose morphisms are inclusion maps. The coset geometry of $G$ with respect to $\left\{G_{a}\right\}_{a \in F}$ is the set

$$
Y=\bigcup_{a \in F} G / G_{a}
$$




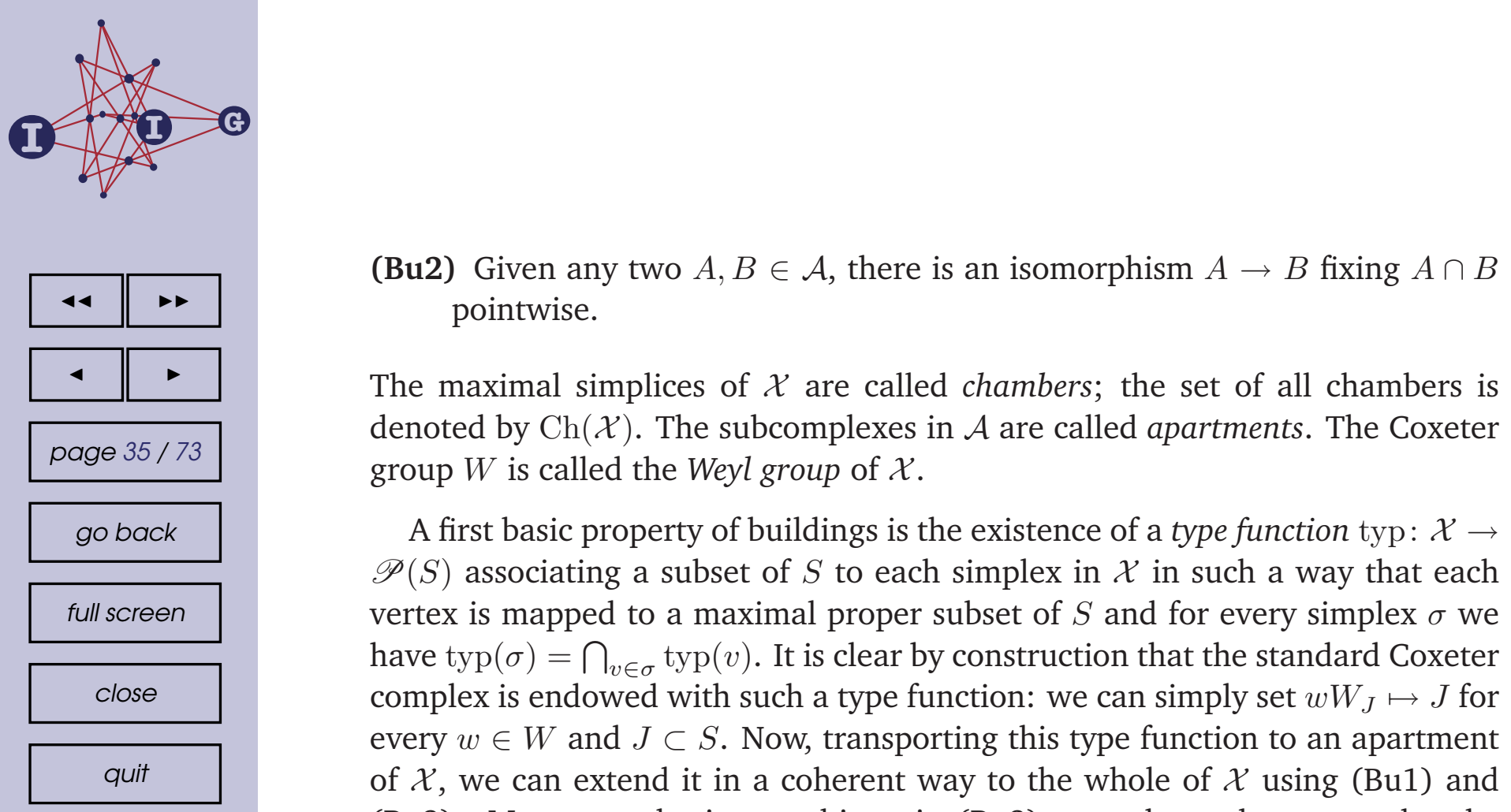

The maximal simplices of $\mathcal{X}$ are called chambers; the set of all chambers is denoted by $\mathrm{Ch}(\mathcal{X})$. The subcomplexes in $\mathcal{A}$ are called apartments. The Coxeter group $W$ is called the Weyl group of $\mathcal{X}$.

A first basic property of buildings is the existence of a type function typ : $\mathcal{X} \rightarrow$ $\mathscr{P}(S)$ associating a subset of $S$ to each simplex in $\mathcal{X}$ in such a way that each vertex is mapped to a maximal proper subset of $S$ and for every simplex $\sigma$ we have $\operatorname{typ}(\sigma)=\bigcap_{v \in \sigma} \operatorname{typ}(v)$. It is clear by construction that the standard Coxeter complex is endowed with such a type function: we can simply set $w W_{J} \mapsto J$ for every $w \in W$ and $J \subset S$. Now, transporting this type function to an apartment of $\mathcal{X}$, we can extend it in a coherent way to the whole of $\mathcal{X}$ using (Bu1) and (Bu2). Moreover, the isomorphisms in (Bu2) may always be assumed to be type-preserving [3, Prop. 4.6]. The type of a chamber is the empty set.

The star of a simplex $\sigma \in \mathcal{X}$ is called a residue. It is itself a building whose apartments are the traces on $\operatorname{St}(\sigma)$ of apartments in $\mathcal{A}$. The type of this building is given by $\left(W_{J}, J\right)$ where $J=\operatorname{typ}(\sigma)$.

\subsection{The Weyl distance}

An important feature about buildings is that the set of chambers is endowed with a so-called Weyl distance. Given a Coxeter system $(W, S)$ and a set $C$, a map $\delta: C \times C \rightarrow W$ is called a Weyl distance if it satisfies the following conditions, where $x, y \in C$ and $w=\delta(x, y)$ :

(WD1) $w=1$ if and only if $x=y$.

(WD2) Given $z \in C$ such that $\delta(y, z)=s \in S$, we have $\delta(x, z) \in\{w, w s\}$; furthermore, if $\ell(w s)>\ell(w)$, then $\delta(x, z)=w s$.

(WD3) Given $s \in S$, there exists $z \in C$ such that $\delta(y, z)=s$ and $\delta(x, z)=w s$.

As we have seen above, the set $\operatorname{Ch}(A)$ of chambers in any apartment of a building $\mathcal{X}$ of type $(W, S)$ can be identified with $W$. Consider the map

$$
\delta_{W}: W \times W \rightarrow W:(x, y) \mapsto x^{-1} y .
$$

It is immediate to check that $\delta_{W}$ is a Weyl distance. Note moreover that the composite map $\ell \circ \delta_{W}: W \times W \rightarrow W$ is nothing but the (combinatorial) distance in the Cayley graph of $W$ with respect to $S$. Now one can transport the Weyl distance $\delta_{W}$ on $\operatorname{Ch}(A)$ for each apartment $A \in \mathcal{A}$ of $\mathcal{X}$. In view of the axioms 



\section{Part II}

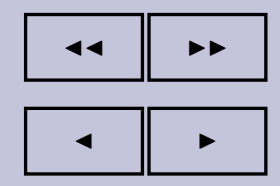

page 37 / 73

go back

full screen

close

quit

ACADEMIA

PRESS

WW

$\widehat{\underline{\text { IIIIII }}}$

UNIVERSITEIT

GENT

\section{Group actions on buildings and associated structure results}

\section{First structure results from actions on buildings}

It is an old matter in group theory to try to obtain a presentation for a group that acts naturally on a space by preserving some structure, e.g. of topological or geometric nature. For example, in the case of a group $\Gamma$ acting by homeomorphisms on an arcwise connected and simply connected topological space $X$ possessing an arcwise connected open subset $U$ such that $\Gamma \cdot U=X$, a precise presentation for $\Gamma$ is given in [55]. An interesting special case is when the $\Gamma$ action is proper and totally discontinuous and $\bar{U}$ is compact: in that case (under some mild extra condition) the given presentation of $\Gamma$ turns out to be finite. This is especially relevant to algebraic topology: the fundamental group of a topological space has a natural action on the universal cover, which is of course simply connected. Thus this method provides a way of obtaining presentations for fundamental groups.

This circle of ideas also lies behind Bass-Serre theory, which characterizes group amalgams in terms of actions on trees. Very early on, Tits realized that these ideas could be efficiently used in the context of buildings (recall that trees are indeed special examples of buildings!). This is what we want to explain in this section.

\subsection{Covering theory for partially ordered sets}

We now describe a very flexible method in the spirit of the ideas described above, which applies in particular to all coset geometries. One of the goals is to make sense of a notion of simple-connectedness for posets in such a way that, under suitable hypotheses, the coset geometry $(Y, \leqslant)$ is simply connected if and only if $G=\lim G_{a}$. We follow [89]; see also [71, Ch. 3].

We consider the category $\mathcal{O}$ whose objects are posets and whose morphisms are non-decreasing maps which are descending bijections. In other words, a nondecreasing map $f:(A, \leqslant) \rightarrow(B, \leqslant)$ is a morphism of $\mathcal{O}$ if and only if for any $a \in A$, the appropriate restriction of $f$ induces a one-to-one map

$$
\{x \in A \mid x \leqslant a\} \rightarrow\{y \in B \mid y \leqslant f(a)\} .
$$





\subsection{Buildings are simply connected}

Let now $(W, S)$ be a Coxeter system and $(\mathcal{X}, \mathcal{A})$ be a building of type $(W, S)$. Let $\mathcal{S}_{2}$ be the set of all subsets $J$ (possibly empty) of $S$ of cardinality at most 2 and such that $W_{J}=\langle J\rangle$ is finite. Let

$$
|\mathcal{X}|_{2}=\left\{\sigma \in \mathcal{X} \mid \sigma \text { is a simplex of type } J \text { for some } J \in \mathcal{S}_{2}\right\},
$$

ordered by inclusion.

Since by definition, the group $W$ is the inductive limit of the system $\left\{W_{J}\right\}_{J \in \mathcal{S}_{2}}$, it follows from Proposition 5.1 that the poset realization $|W|_{2}$ of an apartment of type $(W, S)$ is simply connected. Consequently, we obtain:

Proposition 5.2. The poset realization $|\mathcal{X}|_{2}$ is simply connected.

Proof. Let $f: E \rightarrow|\mathcal{X}|_{2}$ be a covering. We must show that there exists a morphism $h:|\mathcal{X}|_{2} \rightarrow E$ such that $f \cap h=$ id. Let $\sigma_{0}$ be a base chamber in $|\mathcal{X}|_{2}$ and choose $\sigma_{1} \in f^{-1}\left(\sigma_{0}\right)$. Given any $\tau \in|\mathcal{X}|_{2}$, there exists by (Bu1) an apartment $A$ containing both $\sigma$ and $\tau$. Since $|A|_{2}$ is simply connected, one deduces, by considering the restriction of $f$ to the connected component of $f^{-1}\left(|A|_{2}\right)$ containing $\sigma_{1}$, that there exists a morphism $h_{A}: A \rightarrow f^{-1}(A)$ such that $f \circ h_{A}=\operatorname{id}_{|A|_{2}}$ and $h_{A}\left(\sigma_{0}\right)=\sigma_{1}$. In view of (Bu2) and the uniqueness of path-liftings, it follows that for any other apartment $B$ containing $\sigma_{0}$, we have $\left.h_{A}\right|_{A \cap B}=\left.h_{B}\right|_{A \cap B}$. In particular $h_{A}(\tau)$ does not depend on the choice of the apartment $A$. Set $h(\tau)=h_{A}(\tau)$. Now one verifies easily that the map $h:|\mathcal{X}|_{2} \rightarrow E$ is a morphism and the equality $f \circ h=\mathrm{id}$ follows by construction.

One immediately deduces a decomposition as amalgamated sum for groups acting chamber-transitively on buildings. Indeed, a chamber is obvisouly simply connected and if the action is type-preserving and chamber-transitive, then any chamber is automatically a fundamental domain. Thus Propositions 5.1 and 5.2 apply. For example, if $G$ is a group with a BN-pair $(B, N, S)$, then $G$ is the amalgamated sum of the standard parabolic subgroups of type $J$ for $J \in \mathcal{S}_{2}$.

\subsection{Applications to root group data}

Let us now come back to a group $G$ endowed with a root group datum $\left\{U_{\alpha}\right\}_{\alpha \in \Phi}$ of type $E=(\mathbf{B}, \Phi)$, whose Weyl group is denoted by $W$. We let $\left(X_{-}, \delta_{-}\right)$be the building associated with the negative BN-pair $\left(B_{-}, N, S\right)$ of $G$. Our present goal is to apply the technology we have just described to study the $U_{+}$-action on $X_{-}$. 
We first recall the existence of an order $\leqslant$ on $W$ defined as follows:

$$
z \leqslant w \Longleftrightarrow \ell(w)=\ell(z)+\ell\left(z^{-1} w\right)
$$

This is called the Bruhat ordering of $W$. Using the solution of the word problem in Coxeter groups, this is seen to be equivalent to the existence of a reduced word $s_{1} \cdots s_{n}$ representing $w$ as a product of elements of $S$, such that $z=$ $s_{1} \cdots s_{j}$ for some $j \leqslant n$ (or $z=1$ ).

Now, for each $w \in W$, we consider the following subgroup of $U_{+}$:

$$
U_{w}=\left\langle U_{\gamma} \mid \gamma \in \Phi_{w^{-1}}\right\rangle
$$

Using Lemma 1.5, it is easily seen that if $z \leqslant w$, then $U_{z} \leqslant U_{w}$ for all $z, w \in W$. In other words, the system $\left\{U_{w}\right\}_{w \in W}$ is an inductive system of subgroups. As we will see in the sequel, the following result and its proof have many useful consequences concerning the structure of $G$ :

Theorem 5.3. The group $U_{+}$is isomorphic to $\underset{\lim }{\longrightarrow} U_{w}$.

Proof. Let $\widetilde{U}=\lim _{w} U_{w}$. Denote by $\widetilde{U}_{w}$ the canonical image of $U_{w}$ in $\widetilde{U}$ and by $\pi: \widetilde{U} \rightarrow U_{+}$the canonical homomorphism. Consider the set $\widetilde{X}$ consisting of all ordered pairs $\left(u \widetilde{U}_{w}, w W_{J}\right)$ such that $u \in \widetilde{U}, w \in W$, and $J \in \mathcal{S}_{2}$ is such that $w$ is maximal in $w W_{J}$ for the Bruhat ordering. Equivalently, the latter condition means that $w$ is of maximal length in $w W_{J}$; it is a well known fact that there is such a unique element [14, Ch. IV, §1, Exerc. 3].

We define a partial order $\leqslant$ on $\widetilde{X}$ as follows:

$$
\left(u \widetilde{U}_{w}, w W_{I}\right) \leqslant\left(v \widetilde{U}_{z}, z W_{J}\right) \Longleftrightarrow w W_{I} \supset z W_{J} \text { and } v^{-1} u \in \widetilde{U}_{w} .
$$

The condition $w W_{I} \supset z W_{J}$ implies $z \in w W_{I}$ and hence $z \leqslant w$ and $U_{z} \subset U_{w}$. Thus the order $\leqslant$ is well-defined. Obviously there is an order-preserving action of $\widetilde{U}$ on $\widetilde{X}$ defined by $g:\left(u \widetilde{U}_{w}, w W_{I}\right) \mapsto\left(g u \widetilde{U}_{w}, w W_{I}\right)$.

Let now $X_{-}$be the negative building of $G$, namely the building associated with the BN-pair $\left(G, B_{-}, S\right)$ as in Theorem 4.1. Consider the map

$$
\nu: \widetilde{X} \rightarrow\left|X_{-}\right|_{2}:\left(u \widetilde{U}_{w}, w W_{I}\right) \mapsto \pi(u) w P_{I}^{-},
$$

where $P_{I}^{-}$denotes the standard negative parabolic subgroup of type $I$.

The essential points are that $\widetilde{X}$ is connected and $\nu$ is a covering map. The verification of these points is slightly technical but straightforward; details may be found in [71, Th. 3.5.2]. Then it follows from the covering theory of posets 
(see Section 5.1) that $\nu$ is an isomorphism. Moreover $\nu$ is clearly $\pi$-equivariant by construction.

Let us now compare some point-stabilizers in $\widetilde{X}$ and $\left|X_{-}\right|_{2}$. For $w \in W$, we have clearly

$$
\operatorname{Stab}_{\widetilde{U}}\left(\widetilde{U}_{w}, w\right)=\widetilde{U}_{w}
$$

On the other hand, we have $\nu\left(\widetilde{U}_{w}, w\right)=w B_{-}$and

$$
\begin{aligned}
\operatorname{Stab}_{U_{+}}\left(w B_{-}\right) & =\left\{u \in U_{+} \mid u w B_{-}=w B_{-}\right\} \\
& =\left\{u \in U_{+} \mid w^{-1} u w B_{-}=B_{-}\right\} \\
& =U_{+} \cap w B_{-} w^{-1} .
\end{aligned}
$$

From these facts, it follows clearly that

$$
\pi^{-1}\left(U_{+} \cap w B_{-} w^{-1}\right)=\widetilde{U}_{w} .
$$

In particular, for $w=1$ we get $\pi^{-1}\left(U_{+} \cap w B_{-} w^{-1}\right)=\{1\}$ from which it follows that $\pi$ is injective.

Corollary 5.4. We have the following:

(i) For each $w \in W$, we have $U_{+} \cap w B_{-} w^{-1}=U_{w}$. In particular $U_{+} \cap B_{-}=$ $\{1\}$.

(ii) $B_{+} \cap B_{-}=T$.

(iii) We have $U_{-\alpha} \not \subset U_{+}$for each $\alpha \in \Pi$. In particular, the system $\left\{U_{-\alpha}\right\}_{\alpha \in \Phi}$ is a root group datum of type $E$ for $G$ and $\left(B_{+}, N, S\right)$ is a BN-pair.

(iv) We have $T=\bigcap_{\alpha \in \Phi} N_{G}\left(U_{\alpha}\right)$.

Proof. (i) The first assertion follows by transforming (5.1) under $\pi$. The second assertion is the special case of the first one with $w=1$.

(ii) Consider $g=t u \in B_{+}=T . U_{+}$and suppose that $g \in B_{-}=T . U_{-}$. Then $u \in t^{-1} B_{-}=B_{-}$hence $u=1$ by (i), whence $g \in T$ as desired.

(iii) The fact that $U_{-\alpha} \not \subset U_{+}$follows from (i). The second assertion becomes then clear. In particular, we may apply Theorem 4.1 and its corollary. This shows that $\left(B_{+}, N, S\right)$ is indeed a BN-pair for $G$ and that $B_{+}=N_{G}\left(U_{+}\right)$.

(iv) Let $\widetilde{T}=\bigcap_{\alpha \in \Phi} N_{G}\left(U_{\alpha}\right)$. The inclusion $T \subset \widetilde{T}$ follows from the definitions. Note that $\widetilde{T} \subset N_{G}\left(U_{+}\right) \cap N_{G}\left(U_{-}\right)$. By Corollary 4.2 and (iii), we obtain $\widetilde{T} \subset B_{+} \cap B_{-}$. Thus $\widetilde{T} \subset T$ by (ii). 


\subsection{Relationship between the positive and the negative BN- pairs}

The fact that the positive and negative BN-pairs of $G$ have the subgroup $N$ in common is not coincidental. In fact there is a tight relationship between these two BN-pairs, more precisely described by the following:

Proposition 5.5. The following assertions, as well as similar assertions with + and - interchanged, hold:

(i) For all $w \in W$ and $s \in S$ such that $\ell(w s)<\ell(w)$, we have

$$
B_{+} w B_{-} s B_{-}=B_{+} w s B_{-} .
$$

(ii) For each $s \in S$, we have $B_{+} s \cap B_{-}=\varnothing$.

(iii) One has a Birkhoff decomposition, namely the map

$$
W \rightarrow B_{+} \backslash G / B_{-}: w \mapsto B_{+} w B_{-}
$$

is bijective.

Proof. (i) This is established by considerations similar to those used in the proof of Theorem 4.1.

(ii) Assume that $n=b . b^{\prime}$ for some $b \in B_{+}, b^{\prime} \in B_{-}$and $n \in N$ such that $n . T=s \in S$. Let $\alpha \in \Pi$ such that $s=r_{\alpha}$. We have $U_{\alpha}=n U_{-\alpha} n^{-1}$ hence

$$
U_{\alpha}{ }^{b}={ }^{b^{\prime}} U_{-\alpha} .
$$

Since $b \in B_{+}$normalizes $U_{+}$, the group $U_{\alpha}{ }^{b}$ is contained in $U_{+}$. Similarly, we have ${ }^{b^{\prime}} U_{-\alpha} \subset U_{-}$and the equality above shows that $U_{\alpha} \subset{ }^{b}\left(U_{+} \cap U_{-}\right)$. By Corollary 5.3, we have $U_{+} \cap U_{-}=\{1\}$. This yields $U_{\alpha}=\{1\}$ which violates (RGD0). Hence (ii) is proven.

(iii) This is deduced from (i) and (ii) in a similar way as the Bruhat decomposition is obtained from the axioms of BN-pairs. Details may be found in [1, Lemma 1].

Using this result, we may now answer the question: when are $B_{+}$and $B_{-}$ conjugate in $G$ ?

Corollary 5.6. The groups $B_{+}$and $B_{-}$are conjugate in $G$ if and only if $W$ is finite. 










Theorem 6.8. Let $G$ be a group with finite center, endowed with a root group datum $\left\{U_{\alpha}\right\}_{\alpha \in \Phi}$ with $\Phi$ reduced such that $U_{\alpha}$ is finite for each $\alpha \in \Pi$ and that

$$
\sum_{w \in W}(1 / q)^{\ell(w)}<\infty
$$

where $q=\min _{\alpha \in \Pi}\left|U_{\alpha}\right|$ (a sufficient condition is: $q>|S|$ ). Then $U_{+}$is a lattice in $G_{-}$and $G$ is a lattice in $G_{+} \times G_{-}$.

Proof. Let us first consider the group $U_{+}$. By the Birkhoff decomposition $G$ is the disjoint union of subsets of the form $U_{+} \cdot w \cdot B_{-}$where $w$ runs over $W$. This means that the set $\mathcal{C}=\left\{w \cdot B_{-} \mid w \in W\right\}$ is a set of representatives of the $U_{+}$-orbits in $\mathrm{Ch}\left(X_{-}\right)$. By Corollary 5.3(i), we have

$$
\operatorname{Stab}_{U_{+}}\left(w \cdot B_{-}\right)=U_{+} \cap w B_{-} w^{-1}=U_{w} .
$$

By the proposition (see also Proposition 6.1(iv)), the group $U_{+}$is a lattice in $G_{-}$ if and only if $\sum_{w \in W} \frac{1}{\left|U_{w}\right|}<\infty$.

Let us now consider the action of $G$ on $X=X_{+} \times X_{-}$. The product $X$ is a building of type $(W, S)$. Its chamber set $\mathrm{Ch}(X)$ is $\operatorname{Ch}\left(X_{+}\right) \times \operatorname{Ch}\left(X_{-}\right)$. The $G$-action on $X$ preserves the Weyl codistance. Moreover, by the Birkhoff decomposition, it is Weyl co-transitive in the following sense: for any $x, x^{\prime} \in \mathrm{Ch}\left(X_{+}\right)$ and $y, y^{\prime} \in \mathrm{Ch}\left(X_{-}\right)$such that $\delta^{*}(x, y)=\delta^{*}\left(x^{\prime}, y^{\prime}\right)$ there exists $g \in G$ such that $(g . x, g . y)=\left(x^{\prime}, y^{\prime}\right)$. Therefore, it follows that the set $\left\{\left(B_{+}, w \cdot B_{-}\right) \mid w \in W\right\}$ is a set of representatives for the $G$-orbits in $\mathrm{Ch}(X)$. Moreover we have

$$
\operatorname{Stab}_{G}\left(B_{+}, w \cdot B_{-}\right)=B_{+} \cap w B_{-} w^{-1}=T \cdot U_{w}
$$

by Corollary 5.3. Combining Corollary 5.6 with [93, Th. 1], we see that the quotient $T / Z(G)$ is finite, hence so is $T$ because $Z(G)$ is finite by hypothesis. It follows again from the proposition that $G$ is a lattice in $G_{+} \times G_{-}$if and only if $\sum_{w \in W} \frac{1}{\left|U_{w}\right|}<\infty$.

It remains to evaluate the sum $z=\sum_{w \in W} \frac{1}{\left|U_{w}\right|}$. In view of Lemma 5.5, we have $\left|U_{w}\right| \geqslant q^{\ell(w)}$, where $q=\min _{\alpha \in \Pi}\left|U_{\alpha}\right|$. Therefore $z \leqslant \sum_{w \in W}(1 / q)^{\ell(w)}$ as desired.

Note that $\sum_{w \in W} x^{\ell(w)}=\sum_{n \geqslant 0}|W(n)| x^{n}$, where

$$
W(n)=\{w \in W \mid \ell(w)=n\} .
$$

Since we have $|W(n)| \leqslant|S|^{n}$, the condition $q>|S|$ is clearly sufficient for $\sum_{w \in W}(1 / q)^{\ell(w)}$ to converge. 
For the theory of lattices in Lie groups we refer to [67], and for the more advanced and specific theory of lattices in semisimple Lie groups we refer to [56]. These references are the guidelines for the study of lattices arising from the theory of root data with finite root groups as below, at least for the part of the study which relies on analogies with arithmetic groups [70].

\section{Simplicity results}

\subsection{Tits' transitivity lemma}

It is an elementary fact on permutation groups that if a group $G$ acts transitively and primitively on a set $X$ (e.g. $G$ is 2 -transitive), then any normal subgroup of $G$ acts either trivially or transitively. If a group $G$ has a BN-pair, it is not quite true that its action on the chambers of the corresponding building is primitive, but it is indeed true that a chamber-stabilizer has very few over-groups: as mentioned in Section 4.1.1, any subgroup containing $B$ is a standard parabolic subgroup. This should shed some light upon the following:

Lemma 7.1. (Tits' transitivity lemma) Let $G$ be a group with a $B N$-pair $(B, N, S)$ and $X$ be the associated building and $W$ be the Weyl group. If the Coxeter system $(W, S)$ is irreducible, then any normal subgroup of $G$ acts either trivially or transitively on $\mathrm{Ch}(X)$.

Proof. See [83, Prop. 2.5] or [14, Ch. IV, §2, Lemma 2].

In fact, this very useful result might be seen as a variant of a previously known and quite classical theme, according to which groups admitting a sufficiently transitive action on a set shall be submitted to strong restrictions concerning their normal subgroups. To be more precise, we need to introduce some further notions (they will be useful — and still relevant to simplicitywhen discussing some local actions on trees): the action of a group $G$ on a set $X$ is called quasi-primitive if $G$, as well as any non-trivial normal subgroup of $G$, acts transitively on $X$. This is the case if the action is primitive, namely if the only equivalence relations on $X$ which are compatible with the $G$-action are the trivial ones; note that primitivity itself is implied by 2-transitivity. In fact, denoting by $G^{\dagger}$ the subgroup of $G$ (acting on $X$ ) generated by the stabilizers of the various elements $x$ in $X$, we have the following implications:

$G$ acts 2-transitively on $X$

$\Longrightarrow G$ acts primitively on $X$

$\Longrightarrow G$ acts quasi-primitively on $X$, 



for any open normal subgroup $N$, we deduce from [39, Prop. 1.2(iii)] that

$$
[G, G]=\overline{\left(\prod_{i=1}^{d}\left[h_{i}, G\right]\right)^{* f(d)}}
$$

Since the map $G \rightarrow G: g \mapsto\left[h_{i}, g\right]$ is continuous and $G$ is compact, the set $\left[h_{i}, G\right]$ is closed in $G$. Hence the big product in the right-hand side of the latter equation is closed and we obtain

$$
[G, G]=\left(\prod_{i=1}^{d}\left[h_{i}, G\right]\right)^{* f(d)} .
$$

Since $H$ is normal, we have $\left[h_{i}, G\right] \subset H$ for each $i$, from which we finally deduce that $[G, G] \subset H$. Since we have $G=H .[G, G]$ by assumption, it finally follows that $G=H$ as desired.

At this stage, we note that Kac-Moody groups over finite fields provide, through their geometric completions, intriguing topological groups. Indeed, they are often abstractly simple, locally pro- $p$ and share further (combinatorial) properties with adjoint simple algebraic groups over local fields of positive characteristic. This is a probably non-exhaustive list of arguments supporting the analogy with classical matrix groups, but we also saw that the maximal compact subgroups of some of them contain finite index subgroups which are Golod-Shafarevich and hence contain free pro- $p$ subgroups. It would be interesting to provide further arguments supporting and/or disproving this analogy, from the point of view of representation theory for instance.

\subsection{Weyl transitivity of normal subgroups}

The previous simplicity results deal only with the topological completions. No such general simplicity results should be expected for the uncomplete group $G$. Indeed, recall from Section 2.7 that the group $G=\mathrm{SL}_{n}\left(k\left[t, t^{-1}\right]\right)$ possesses a root group datum, but it is far from simple in view of the existence of evaluation homomorphisms. However, in the context of root group data, Theorem 7.2 may be used to obtain a strengthening of Tits' transitivity lemma.

Before stating it, we introduce the following definition: a group $G$, acting on a building $X$ with Weyl distance $\delta$, is called Weyl transitive if for any elements $x, y, x^{\prime}, y^{\prime} \in \operatorname{Ch}(X)$ such that $\delta(x, y)=\delta\left(x^{\prime}, y^{\prime}\right)$, there exists $g \in G$ such that $(g . x, g \cdot y)=\left(x^{\prime}, y^{\prime}\right)$. It is an immediate consequence of the Bruhat decomposition that if $G$ has a BN-pair, then $G$ is Weyl transitive on the associated building. The following result is a straightforward consequence of Theorem 7.2: 





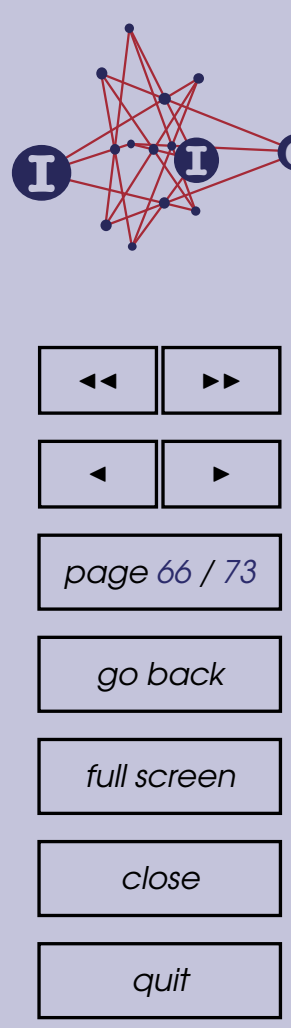

nilpotent. This is because the rank one group $X_{\alpha}=\left\langle U_{\alpha} \cup U_{-\alpha}\right\rangle$ is never nilpotent (see Corollary 5.6); in fact $X_{\alpha}$ is quasisimple unless it is finite of very small order. However, the residual nilpotency of $U_{+}$is delicate to establish. The way it is done in [63] is by realizing the inductive system of rank two groups of $\mathscr{D}$ in a certain large group which is known to possess a root group datum (mostly the latter group is a split Kac-Moody group). This allows to embed $U_{+}$in some unipotent radical of this larger group. Now the latter group is residually nilpotent as a consequence of Proposition 6.3, hence so is $U_{+}$.

(2) The article [24] quoted above is concerned with the isomorphism problem for groups endowed with locally finite root group data, while [22] deals with the case of split Kac-Moody groups. None of these references makes the assumption that the Weyl group is 2-spherical.

(3) The only reason for the assumption that $G\left(\mathscr{D}_{1}\right)$ is infinite and $\mathscr{D}_{1}$ of irreducible type in Theorem 8.2(ii) is to avoid the exceptional isomorphisms between small finite Chevalley groups. Of course, the conclusions of that theorem are known to hold for all sufficiently large finite Chevalley groups: this is all classical, see [80].

\section{References}

[1] P. Abramenko, Twin buildings and applications to S-arithmetic groups, Lecture Notes in Math., vol. 1641, Springer-Verlag, 1996.

[2] - Finiteness properties of groups acting on twin buildings, in Groups: topological, combinatorial and arithmetic aspects (ed. T. Müller), London Math. Soc. Lecture Note Ser., vol. 311, Cambridge Univ. Press, 2004, pp. 21-26.

[3] P. Abramenko and K. Brown, Buildings. Theory and applications, Grad. Texts in Math., vol. 248, Springer, New York, 2008.

[4] , Transitivity properties for group actions on buildings, J. Group Theory 10 (2007), 267-277.

[5] P. Abramenko and B. Mühlherr, Présentations de certaines $B N$-paires jumelées comme sommes amalgamées, C. R. Acad. Sci. Paris Sér. I Math. 325 (1997), 701-706.

[6] U. Bader and Y. Shalom, Factor and normal subgroup theorems for lattices in products of groups, Invent. Math. 163 (2006), 415-454. 
[7] N. Bardy, Systèmes de racines infinis, Mém. Soc. Math. Fr. 65 (1996).

[8] H. Behr, Arithmetic groups over function fields. I. A complete characterization of finitely generated and finitely presented arithmetic subgroups of reductive algebraic groups, J. Reine Angew. Math. 495 (1998), 79-118.

[9] A. Borel, Groupes linéaires algébriques, Ann. of Math. 64 (1956), 20-82.

[10] _ Linear algebraic groups, Grad. Texts in Math., vol. 126, SpringerVerlag, 1991, 2nd edition.

[11] A. Borel and J. Tits, Groupes réductifs, Publ. Math. Inst. Hautes Études Sci. 27 (1965), 55-150.

[12] _ Homomorphismes "abstraits" de groupes algébriques simples, Ann. of Math. 97 (1973), 499-571.

[13] N. Bourbaki, Éléments de mathématique. Intégration VII-VIII, Springer, 2007.

[14] Éléments de mathématique. Lie IV-VI, Springer, 2007.

[15] M. Bourdon, Sur les immeubles fuchsiens et leur type de quasi-isométrie, Ergodic Theory Dynam. Systems 20 (2000), 343-364.

[16] F. Bruhat, Représentations induites des groupes de Lie semi-simples complexes, C. R. Math. Acad. Sci. Paris 238 (1954), 437-439.

[17] F. Bruhat and J. Tits, Groupes réductifs sur un corps local. I: Données radicielles valuées, Publ. Math. Inst. Hautes Études Sci. 41 (1972), 5-251.

[18] Groupes réductifs sur un corps local. II. Schémas en groupes. Existence d'une donnée radicielle valuée, Publ. Math. Inst. Hautes Études Sci. 60 (1984), 197-376.

[19] M. Burger and S. Mozes, Groups acting on trees: from local to global structure, Publ. Math. Inst. Hautes Études Sci. 92 (2000), 113-150.

[20] , Lattices in product of trees, Publ. Math. Inst. Hautes Études Sci. (2000), no. 92, 151-194.

[21] P.-E. Caprace, On 2-spherical Kac-Moody groups and their central extensions, Forum Math. 19 (2007), 763-781.

[22] _ "Abstract" homomorphisms of split Kac-Moody groups, Mem. Amer. Math. Soc. 198 (2009). 
[23] _ A uniform bound on the nilpotency degree of certain subalgebras of Kac-Moody algebras, J. Algebra 317 (2007), 867-876.

[24] P.-E. Caprace and B. Mühlherr, Isomorphisms of Kac-Moody groups which preserve bounded subgroups, Adv. Math. 206 (2006), 250-278.

[25] P.-E. Caprace and B. Rémy, Simplicity and superrigidity of Kac-Moody lattices, Invent. Math. 176 (2009), 169-221.

[26] L. Carbone, M. Ershov and G. Ritter, Abstract simplicity of complete Kac-Moody groups over finite fields, J. Pure Appl. Algebra 212 (2008), 2147-2162.

[27] L. Carbone and H. Garland, Lattices in Kac-Moody groups, Math. Res. Lett. 6 (1999), 439-448.

[28] C. Chevalley, Sur certains groupes simples, Tôhoku Math. J. 7 (1955), 14-66.

[29] Classification des groupes algébriques semi-simples, SpringerVerlag, 2005, collected works. vol. 3, Edited and with a preface by P. Cartier, With the collaboration of P. Cartier, A. Grothendieck and M. Lazard.

[30] C. W. Curtis, Central extensions of groups of Lie type, J. Reine Angew. Math. 220 (1965), 174-185.

[31] M. Davis, The geometry and topology of Coxeter groups, London Math. Soc. Monogr. Ser., vol. 32, Princeton University Press, 2008.

[32] P. de la Harpe and A. Valette, La propriété $(T)$ de Kazhdan pour les groupes localement compacts, Astérisque, Soc. Math. de France. With an appendix by M. Burger.

[33] T. De Medts and Y. Segev, A course on Moufang sets, Innov. Incidence Geom. 9 (2009), 79-122.

[34] T. De Medts and K. Tent, Central extensions of rank 2 groups and applications, Forum Math. 21 (2009), 1-21.

[35] T. De Medts and R. Weiss, Moufang sets and Jordan division algebras, Math. Ann. 335 (2006), 415-433.

[36] M. Demazure and A. Grothendieck, Schémas en groupes. I: Propriétés générales des schémas en groupes, Séminaire de Géométrie Algébrique du Bois Marie 1962/64 (SGA 3). Dirigé par M. Demazure et A. Grothendieck. Lecture Notes in Math., vol. 151, Springer-Verlag, 1970. 
[37] A. Devillers and B. Mühlherr, On the simple connectedness of certain subsets of buildings, Forum Math. 19 (2007), no. 6, 955-970.

[38] J. Dieudonné, La géométrie des groupes classiques, troisième édition, Ergeb. Math. Grenzgeb., vol. 5, Springer-Verlag, 1971.

[39] J. Dixon, M. du Sautoy, A. Mann and D. Segal, Analytic pro-p groups, Cambridge Stud. Adv. Math., vol. 61, Cambridge University Press, 1999, second edition.

[40] J. Dymara and T. Januszkiewicz, Cohomology of buildings and their automorphism groups, Invent. Math. 150 (2002), 579-627.

[41] M. Ershov, Golod-Shafarevich groups with property (T) and Kac-Moody groups, Duke Math. J. 145 (2008), 309-339.

[42] H. Freudenthal, Lie groups in the foundations of geometry, Adv. Math. 1 (1964), 145-190.

[43] P. Gille, Le problème de Kneser-Tits (novembre 2007), séminaire Bourbaki, exposé 985 .

[44] M. Hall, The theory of groups, Chelsea Publishing Co., 1976, reprinting of the 1968 edition.

[45] J.-Y. Hée, Construction de groupes tordus en théorie de Kac-Moody, $C$. R. Acad. Sci. Paris Sér. I Math. 310 (1990), 77-80.

[46] _ Système de racines sur un anneau commutatif totalement ordonné, Geom. Dedicata 37 (1991), 65-102.

[47] C. Hering, W. Kantor and G. Seitz, Finite groups with a split $B N$-pair of rank 1. I, J. Algebra 20 (1972), 435-475.

[48] E. Hewitt and K. Ross, Abstract harmonic analysis. Vol. I, Grundlehren Math. Wiss., vol. 115, Springer-Verlag, 1979.

[49] N. Iwahori and H. Matsumoto, On some Bruhat decomposition and the structure of the Hecke rings of $p$-adic Chevalley groups, Publ. Math. Inst. Hautes Études Sci. 25 (1965), 5-48.

[50] V. Kac, Infinite dimensional Lie algebras, Cambridge University Press, 1990, 3rd edition.

[51] V. Kac and D. Peterson, Defining relations of certain infinite-dimensional groups, Astérisque (1985), no. Hors Série, 165-208, The mathematical heritage of Élie Cartan (Lyon, 1984). 
[52] M.-A. Knus, A. Merkurjev, M. Rost and J.-P. Tignol, The book of involutions, Amer. Math. Soc. Colloq. Publ., vol. 44, American Mathematical Society, 1998, with a preface in French by J. Tits.

[53] D. Krammer, The conjugacy problem for Coxeter groups, Groups Geom. Dyn. 3 (2009), 71-171.

[54] S. Kumar, Kac-Moody groups, their flag varieties and representation theory, Progr. Math., vol. 204, Birkhäuser, 2002.

[55] A. Macbeath, Groups of homeomorphisms of a simply connected space, Ann. of Math. 79 (1964), 473-488.

[56] G. Margulis, Discrete subgroups of semisimple Lie groups, Ergeb. Math. Grenzgeb., vol. 17, Springer-Verlag, 1991.

[57] O. Mathieu, Formules de caractères pour les algèbres de Kac-Moody générales, number 159-160 in Astérisque, Soc. Math. de France, 1988.

[58] R. Moody, A simplicity theorem for Chevalley groups defined by generalized Cartan matrices (1982), preprint.

[59] R. Moody and A. Pianzola, On infinite root systems, Trans. Amer. Math. Soc. 315 (1989), 661-696.

[60] Lie algebras with triangular decompositions, Canad. Math. Soc. Ser. Monogr. Adv. Texts, Wiley Interscience, 1995.

[61] R. Moody and K. Teo, Tits' systems with crystallographic Weyl groups, J. Algebra 21 (1972), 178-190.

[62] G. Mostow, Strong rigidity of locally symmetric spaces, Ann. of Math. Stud., vol. 78, Princeton University Press, 1973.

[63] B. Mühlherr, Locally split and locally finite twin buildings of 2-spherical type, J. Reine Angew. Math. 511 (1999), 119-143.

[64] _ Twin buildings, in Tits buildings and the model theory of groups (Würzburg, 2000) (ed. K. Tent), London Math. Soc. Lecture Note Ser., vol. 291, Cambridge Univ. Press, 2002, pp. 103-117.

[65] G. A. Noskov and E. B. Vinberg, Strong Tits alternative for subgroups of Coxeter groups, J. Lie Theory 12 (2002), 259-264.

[66] V. Platonov and A. Rapinchuk, Algebraic groups and number theory, Pure Appl. Math., vol. 139, Academic Press, 1994. 
[67] M. Raghunathan, Discrete subgroups of Lie groups, Ergeb. Math. Grenzgeb., vol. 68, Springer-Verlag, 1972.

[68] D. Rattaggi, Computations in groups acting on a product of trees: normal subgroup structures and quaternion lattices, Ph.D. thesis, ETH Zurich, 2004.

[69] B. Rémy, Construction de réseaux en théorie de Kac-Moody, C. R. Math. Acad. Sc. Paris 329 (1999), 475-478.

[70] Classical and non-linearity properties of Kac-Moody lattices, in Rigidity in Dynamics and Geometry (Newton Institute, Cambridge, 2000) (eds. M. Burger and A. Iozzi), Springer-Verlag, 2002, pp. 391-406.

[71] _ Groupes de Kac-Moody déployés et presque déployés, number 277 in Astérisque, Soc. Math. de France, 2002.

[72] __ Immeubles de Kac-Moody hyperboliques, groupes non isomorphes de même immeuble, Geom. Dedicata 90 (2002), 29-44.

[73] , Integrability of induction cocycles for Kac-Moody groups, Math. Ann. 333 (2005), 29-43.

[74] B. Rémy and M. Ronan, Topological groups of Kac-Moody type, rightangled twinnings and their lattices, Comment. Math. Helv. 81 (2006), 191-219.

[75] M. Ronan, Lectures on buildings, Perspectives in Mathematics, vol. 7, Academic Press, 1989.

[76] D. Segal, Closed subgroups of profinite groups, Proc. London Math. Soc. 81 (2000), 29-54.

[77] J.-P. Serre, Arbres, amalgames, $\mathrm{SL}_{2}$, Soc. Math. de France, 1977, rédigé avec la collaboration de H. Bass, Astérisque, No. 46.

[78] Y. Shalom, Rigidity of commensurators and irreducible lattices, Invent. Math. 141 (2000), 1-54.

[79] T. Springer, Linear algebraic groups, Progr. Math. vol. 9, Birkhauser, 1998, 2nd edition.

[80] R. Steinberg, Lectures on Chevalley groups, mimeographed lecture notes, Yale university, 1968.

[81] J. Tits, Généralisations des groupes projectifs basées sur leurs propriétés de transitivité, Acad. Roy. Belgique. Cl. Sci. Mém. Coll. in $8^{\circ} 27$ (1952), 115 pages. 
[82] _. Sur les analogues algébriques des groupes semi-simples complexes, in Colloque d'algèbre supérieure, Bruxelles, 19-22 décembre 1956, Centre Belge de Recherches Mathématiques, 1957, pp. 261-289.

[83] _ Algebraic and abstract simple groups, Ann. of Math. 80 (1964), $313-329$.

[84] _ Normalisateurs de tores. I. Groupes de Coxeter étendus, J. Algebra 4 (1966), 96-116.

[85] _ Buildings of spherical type and finite BN-pairs, Springer-Verlag, Lecture Notes in Math., vol. 386, Springer-Verlag, New York, Heidelberg, Berlin, 1974.

[86] _ Groupes de Whitehead de groupes algébriques simples sur un corps (d'après V. P. Platonov et al.), in Séminaire Bourbaki, Exp. 505 (1976/77), Lecture Notes in Math., vol. 677, Springer, 1978, pp. 218236.

[87] , A local approach to buildings, in The geometric vein, Springer, 1981, pp. 519-547.

[88] _ Moufang octagons and the Ree groups of type ${ }^{2} F_{4}$, Amer. J. Math. 105 (1983), 539-594.

[89] Ensembles ordonnés, immeubles et sommes amalgamées, Bull. Soc. Math. Belg. Sér. A 38 (1986), 367-387.

[90] _ Immeubles de type affine, in Buildings and the geometry of diagrams (Como, 1984), Lecture Notes in Math., vol. 1181, Springer, 1986, pp. 159-190.

[91] _ Uniqueness and presentation of Kac-Moody groups over fields, J. Algebra 105 (1987), 542-573.

[92] _ Groupes associés aux algèbres de Kac-Moody, Séminaire Bourbaki, vol. 1988/89, Astérisque 177-178 (1989), Exp. No. 700, 7-31.

[93] __ Théorie des groupes, Ann. Collège France 89 (1988/89), 81-96, résumé de cours.

[94] _ Théorie des groupes, Ann. Collège France 90 (1989/90), 87-104, résumé de cours.

[95] _ Twin buildings and groups of Kac-Moody type, in Groups, combinatorics \& geometry (Durham, 1990) (eds. M. Liebeck and J. Saxl), Lecture Note Ser., vol. 165, London Math. Soc., 1992, pp. 249-286. 
